\title{
Oxidative Stress in Genetic Mouse Models of Parkinson's Disease
}

\author{
Mustafa Varçin, Eduard Bentea, Yvette Michotte, and Sophie Sarre \\ Department of Pharmaceutical Chemistry, Drug Analysis and Drug Information, Center for Neurosciences, Faculty of Medicine and \\ Pharmacy, Vrije Universiteit Brussel, Laarbeeklaan 103, 1090 Brussels, Belgium
}

Correspondence should be addressed to Sophie Sarre, ssarre@vub.ac.be

Received 24 February 2012; Revised 12 April 2012; Accepted 12 April 2012

Academic Editor: Krzysztof Ksiazek

Copyright ( $) 2012$ Mustafa Varçin et al. This is an open access article distributed under the Creative Commons Attribution License, which permits unrestricted use, distribution, and reproduction in any medium, provided the original work is properly cited.

\begin{abstract}
There is extensive evidence in Parkinson's disease of a link between oxidative stress and some of the monogenically inherited Parkinson's disease-associated genes. This paper focuses on the importance of this link and potential impact on neuronal function. Basic mechanisms of oxidative stress, the cellular antioxidant machinery, and the main sources of cellular oxidative stress are reviewed. Moreover, attention is given to the complex interaction between oxidative stress and other prominent pathogenic pathways in Parkinson's disease, such as mitochondrial dysfunction and neuroinflammation. Furthermore, an overview of the existing genetic mouse models of Parkinson's disease is given and the evidence of oxidative stress in these models highlighted. Taken into consideration the importance of ageing and environmental factors as a risk for developing Parkinson's disease, geneenvironment interactions in genetically engineered mouse models of Parkinson's disease are also discussed, highlighting the role of oxidative damage in the interplay between genetic makeup, environmental stress, and ageing in Parkinson's disease.
\end{abstract}

\section{Introduction}

Parkinson's disease (PD) is a highly prevalent neurodegenerative disorder, second to Alzheimer's disease in terms of incidence [1]. Its prevalence increases with age, being approximately $1 \%$ in people over the age of 60 and increasing to about $4 \%$ over the age of 85 [2]. It affected about 4.5 million people over the age of 50 in 2005, and by the year 2030, this number will have doubled [3]. The etiology is still unknown [4], but it is hypothesized that it may result from a complex interaction between environmental factors, genetic susceptibility and ageing $[5,6]$.

Diagnosed patients are characterized by motor and nonmotor clinical manifestations. The motor symptoms include resting tremor, bradykinesia, akinesia, muscular rigidity, and a loss of balance [7] and are predominantly attributed to a lack of dopamine (DA) in the striatum, and the resulting dysfunction of the basal ganglia, a cluster of nuclei involved in the initiation and execution of movement $[1,7]$. The nonmotor symptoms include impaired olfaction, sleep disorders, constipation, urinary incontinence, orthostatic hypotension, and various neuropsychiatric manifestations (e.g., depression, hallucinations, dementia), and can appear both before and during the motor symptomatology of PD [7].

The pathological hallmark of PD comprises loss of nigrostriatal dopaminergic neurons in the substantia nigra (SN) pars compacta $(\mathrm{SNc})$ and the presence of insoluble protein inclusions termed Lewy bodies (LBs) and Lewy neurites (LNs), located in either the neuronal cell bodies or neuronal processes, respectively $[2,6,8]$. The major constituent of LBs and LNs is a misfolded version of the protein alpha-synuclein ( $\alpha$-syn) [8]. There are also nondopaminergic neurons affected in $\mathrm{PD}$, leading to more wide-spread neuronal changes that cause a complex and heterogeneous clinical picture [7, 9]. Recently, Braak and colleagues hypothesized a six-stage pathological process in which PD pathology emerges in the olfactory bulb and the dorsal motor nucleus of the vagal nerve and only in later stages extends to the midbrain and other brainstem regions $[10,11]$. Current therapies of $\mathrm{PD}$ are symptomatic, targeting the lack of DA in the striatum with DA replacement strategies. Although these therapies provide symptomatic 
relief at the beginning, they become gradually inefficient as the disease progresses. Therefore, there is an urgent need of therapeutic strategies that can tackle the disease progression [12].

Despite the fact that PD has long been considered as a non-genetic disorder of sporadic origin, research performed during the past decade has led to the identification of genes linked to rare monogenic forms of PD. This resulted in the identification of 16 "PARK" loci, with the autosomal dominant genes SNCA (PARK1/4), and LRRK2 (PARK8), and the autosomal recessive genes parkin (PARK2), DJ-1 (PARK7), and PINK1 (PARK6) being the most common ones $[2,3,5]$. Although monogenic forms account for $<10 \%$ of PD cases, these genes also play a role in the much more common sporadic form of the disease [5]. Unraveling the molecular mechanisms underlying the familial forms of PD will contribute to our understanding of sporadic PD, since both share clinical and neuropathological features [5]. Moreover, several cellular abnormalities which may underlie the neurodegeneration displayed in sporadic PD, such as mitochondrial dysfunction, oxidative stress, excitotoxicity, proteasomal stress, neuroinflammation, and protein aggregation, are also associated with mutations in the familial PD genes $[13,14]$.

Multiple animal models have been developed in order to study the pathogenesis and progression of $\mathrm{PD}$ and to test potential therapeutic strategies [15]. Currently, focus is being put on integrating genetic, environmental, and age-related influences in more relevant preclinical animal models, which can reproduce PD pathology with high fidelity.

Since there is extensive evidence of oxidative stress in the pathogenesis of PD [14, 16-20], and a link between oxidative stress and some of the monogenically inherited PD associated genes has been described, this paper focuses on its importance and potential impact on neuronal function. It is interesting that despite all the evidence for a role of oxidative stress in PD, there have been relatively few studies that have extensively characterized oxidative stress in animal models of $\mathrm{PD}$ [21]. In this paper, an overview is given of oxidative stress findings in monogenic mouse models of PD. In addition, a focus will be put on gene-environment interactions in these mouse models.

\section{Genetic Forms of Parkinson's Disease}

The PD genes can show both recessive and dominant modes of inheritance. The autosomal dominant PD genes include SNCA, leucine-rich repeat kinase 2 (LRRK2), microtubuleassociated protein tau (MAPT), Htr serine peptidase 2 (HtrA2), and glucocerebrosidase (GBA), while the autosomal recessive $\mathrm{PD}$ genes include parkin, $\mathrm{PTEN}$-induced kinase 1 (PINK1), and DJ-1 $[5,22]$. In the context of this paper, only SNCA, parkin, PINK1, and DJ-1 are discussed in further detail.

Studies of PD-linked genes have brought to light several pathways involved in neuronal death in the SNc (protein aggregation, defects in the ubiquitin-proteasomal pathway, impaired defense against oxidative stress, abnormal protein phosphorylation, mitochondrial and lysosomal dysfunction, apoptosis), thus improving our understanding of the more common sporadic form of the disease. "Sporadic" cases may, in fact, be monogenic as well, because mutations may have occurred de novo [5].

2.1. SNCA. The SNCA gene encodes $\alpha$-syn, a small 140 amino acid protein that can be both lipid associated and free in the cytoplasm [23]. It has been shown that $\alpha$-syn promotes PD pathogenesis either by dominantly inherited mutations in the SNCA gene (A30P, A53T, and E46K) or by factors that enhance $\alpha$-syn expression, such as gene multiplication (e.g., duplication, triplication) of the SNCA gene or by SNCA promoter polymorphisms that increase $\alpha$-syn expression [2, 7]. Although the three missense mutations in SNCA are very rare, a normal expression of a mutant form of $\alpha$-syn, or overexpression of the wild-type (WT) $\alpha$-syn, can contribute to the pathogenesis of PD [2]. Furthermore, a clear evidence for an SNCA dosage effect was also revealed in one familial case of PD [2].

The phenotype of patients with SNCA mutations resembles sporadic PD, but with earlier onset and atypical features, including cognitive decline, psychiatric problems, and autonomic dysfunction. The same mutations have been found in sporadic PD patients, suggesting significant frequency of de novo mutations [5].

2.2. Parkin. Parkin is a 465 amino acid E3 ubiquitin ligase, which transfers ubiquitin to target proteins for degradative (i.e., via the proteasome system) or non-degradative (i.e., signaling) purposes. Mutations in parkin account for the majority of early-onset familial PD cases [24]. Loss-offunction of parkin can contribute to the etiology of PD either by disrupting the normal function of the ubiquitin proteasome system (UPS) in the clearance of aggregated proteins or by disabling a mitochondrial protective mechanism mediated by parkin's signaling function, contributing to mitochondrial dysfunction [24].

2.3. PINK1. PINK1 encodes a 581 amino acid serine/threonine kinase that is localized to the mitochondria. Mutations in PINK1 are the second most common cause of autosomal recessive early-onset familial PD after parkin, with most mutations occurring in and disrupting the activity of the kinase domain [18, 24, 25]. As with parkin, loss-of-function of PINK1 leads to decreased mitochondrial protection against oxidative stress, causing enhanced mitochondrial dysfunction [26]. Although the biological function of the PINK1 protein is not fully understood, studies show an important role in the maintenance of mitochondrial function and protection against oxidative stress [27-30].

2.4. DJ-1. DJ-1 encodes a 189 amino acid protein of stilluncertain function. Mutations in DJ-1 represent a rather rare cause of early-onset familial PD [24]. Still, insights into putative roles of DJ-1 brought this protein under investigation, and it seems that DJ-1 is involved alongside parkin and PINK1 in protecting the mitochondria against 
oxidative stress [7]. Interestingly, reduced DJ-1 expression is also associated with proteasome inhibition [23], highlighting an additional role of DJ-1 in the normal function of the UPS. The DJ-1 protein was found to be oxidatively damaged and significantly increased in brains of sporadic PD patients [5].

Patients carrying a mutation in parkin, PINK1, or DJ-1 show marked clinical overlap, generally suffering from early-onset parkinsonism with a slower disease progression, good levodopa response, and early development of motor fluctuations [2].

\section{Oxidative Stress in Parkinson's Disease}

Oxidative stress can be defined as a condition in which the cellular antioxidant defense mechanisms are insufficient to keep the level of reactive oxygen species (ROS) below a toxic threshold [7]. This may be either due to an overproduction of reactive free radicals or to a failure of cell buffering mechanisms [14]. ROS can damage all types of biomolecules, and oxidative damage to nucleic acids, lipids, and proteins can be deleterious [31]. Several of the genes linked to familial forms of $\mathrm{PD}$ appear to be involved in the protection against or in the propagation of oxidative stress $[14,32]$. Furthermore, oxidative stress and mitochondrial dysfunction have been linked to the pathogenesis of PD, ever since exposure to 1-methyl-4-phenyl-1,2,3,6-tetrahydropyridine (MPTP), a complex I inhibitor, was found to induce parkinsonism in humans $[30,33]$. Data from postmortem studies of brains from patients with PD (Table 1) suggest that oxidative stress plays an important role in neuronal degeneration of the dopaminergic nigral neurons [34, 35]. Early and profound loss of glutathione (GSH) levels, a reduction in mitochondrial complex I activity, increased oxidative damage to lipids, proteins, and DNA, increased superoxide dismutase (SOD) activity, and elevated free iron levels in the SN of PD patients have been demonstrated [9, 15, 3539]. Moreover, in vivo observations (Table 1) demonstrated that several markers of oxidative stress are altered in the cerebrospinal fluid (CSF) and blood samples of PD patients $[40,41]$.

Nevertheless, it should be noted that many clinical trials are done in chronically treated PD patients, and drug intake may influence the outcome of the study. For instance, a welldescribed effect of chronic levodopa intake is the elevation of the homocysteine plasma levels, which is a risk factor for various pathological conditions possibly due to homocysteinemediated increase in oxidative stress [47-50]. Furthermore, Buhmann et al. [43] have shown that levodopa monotherapy results in an increase in auto-oxidation and in a decrease of plasma antioxidants with significance for ubiquinol-10, while DA agonist monotherapy was associated with higher alpha-tocopherol levels [43]. Müller and Muhlack [48] have also shown that acute levodopa/carbidopa application reduces free cysteine/glycine levels in plasma and that this decline may be linked to prior appearance of oxidative stress with concomitant consumption of antioxidants like GSH and subsequent conversion of this molecule to oxidized GSH (GSSG) [48]. Therefore, the study design of the clinical trial should be carefully considered when investigating oxidative stress in PD patients.

It is interesting to note that the brain in particular is more vulnerable to oxidative stress and oxidative damage compared to other organs. For instance, the brain consumes more oxygen on a per weight basis under physiological conditions than any other organ, thereby increasing its susceptibility to oxidative stress. Also, the brain contains a relatively low level of antioxidants and free radical scavenging enzymes compared to other tissues [51-53], as well as a high amount of substances, such as phospholipids and unsaturated fatty acids, which are vulnerable to oxidative modifications [33, 54]. Indeed, markers of lipid peroxidation, such as elevated 4-hydroxynonenal (4-HNE) and malondialdehyde (MDA), have been observed in postmortem brain tissue and CSF of PD patients [42, 51]. Moreover, the vulnerability of neurons to oxidative damage, which accumulates in ageing neurons, might also be due to their postmitotic nature [55].

ROS are produced by a number of different pathways, including direct interactions between redox-active metals and oxygen species via reactions such as the Fenton and Haber-Weiss reactions, or by indirect pathways involving activation of enzymes, such as nitric oxide synthase (NOS). All the initial free radical reactions require activation of molecular oxygen [51]. It is important to realize that ROS are being continuously generated in vivo as a result of oxygen metabolism, with about $1-5 \%$ of the oxygen consumed being converted to ROS [30]. The univalent reduction of molecular oxygen leads to the formation of superoxide anion $\left(\mathrm{O}_{2}{ }^{--}\right)$, hydrogen peroxide $\left(\mathrm{H}_{2} \mathrm{O}_{2}\right)$, and hydroxyl radicals. The $\mathrm{O}_{2}{ }^{\bullet-}$ can lead to the generation of hydroxyl radicals via $\mathrm{Fe}^{2+}$ catalyzed Haber-Weiss and Fenton reactions. The generation of reactive nitrogen species (RNS) is due to NOS-mediated conversion of arginine to citrulline and the subsequent generation of nitric oxide $(\mathrm{NO})$, which reacts with $\mathrm{O}_{2}{ }^{--}$to produce peroxynitrite $\left(\mathrm{ONOO}^{-}\right)$[51].

The high concentration of DA in the nigrostriatal pathway is presumed to be an essential determinant for the high vulnerability of dopaminergic cells to oxidative stress. DA itself does not exert direct toxic effects, but toxic intermediates derived from its catabolism [54] may contribute to the oxidative stress pathogenic pathway in PD. The catabolism of DA includes auto-oxidation into toxic DAquinone species, $\mathrm{O}_{2}{ }^{\bullet-}$ and $\mathrm{H}_{2} \mathrm{O}_{2}$, and the enzymatic conversion via monoamine oxidase $\mathrm{B}(\mathrm{MAO}-\mathrm{B})$ into the inert 3,4dihydroxyphenylacetic acid (DOPAC) and $\mathrm{H}_{2} \mathrm{O}_{2}$ [56]. Furthermore, the $\mathrm{H}_{2} \mathrm{O}_{2}$ and $\mathrm{O}_{2}{ }^{--}$produced by DA catabolism can be further converted into highly toxic hydroxyl radicals as described above $[17,36]$. It is important to note that these hydroxyl radical generating pathways are heavily dependent on the presence of iron, which have been found to be elevated in the SN of PD patients [36]. The particular reason for this is not yet understood, but it seems to be related with the age-dependent accumulation of neuromelanin in the nigral neurons [51]. Neuromelanin is a dark-brown pigment that concentrates metal ions, in particular iron, and that makes the nigrostriatal dopaminergic neurons seem dark colored. The age-dependent accumulation of neuromelanin will lead to a higher local concentration of iron in dopaminergic 
TABLE 1: Oxidative stress parameters in Parkinson's disease patients.

\begin{tabular}{|c|c|c|c|c|}
\hline Observation & Marker for oxidative stress & Sampled region & Outcome (versus control) & References \\
\hline \multirow[t]{4}{*}{ In vivo } & MDA & Blood, CSF & $\uparrow$ & {$[40]$} \\
\hline & GSH reductase & Blood & $\uparrow$ & \\
\hline & $\mathrm{Cu} / \mathrm{Zn}-\mathrm{SOD}$ & Blood & $\uparrow$ & \\
\hline & Superoxide & Blood & $\uparrow$ & \\
\hline In vivo & HNE & Plasma, CSF & $\uparrow$ & {$[42]$} \\
\hline \multirow[t]{3}{*}{ In vivo } & Lipoprotein oxidation & Plasma, CSF & $\uparrow$ & {$[43]$} \\
\hline & Protein sulfhydryl groups & Plasma, CSF & $\downarrow$ & \\
\hline & $\alpha$-Tocopherol & CSF & $\downarrow$ & \\
\hline \multirow{3}{*}{ In vivo } & $\mathrm{Cu} / \mathrm{Zn} / \mathrm{Mn}-\mathrm{SOD}$ activity & Skin fibroblasts & $\leftrightarrow$ & {$[44]$} \\
\hline & GPx activity & Skin fibroblasts & $\leftrightarrow$ & \\
\hline & Catalase activity & Skin fibroblasts & $\leftrightarrow$ & \\
\hline \multirow[t]{2}{*}{ In vivo } & Lipid oxidation products & Plasma & $\uparrow$ & {$[19]$} \\
\hline & 8-OHdG & Urine & $\uparrow$ & \\
\hline \multirow[t]{2}{*}{ In vivo } & Markers of oxidative stress & iPSCs & $\uparrow$ & {$[45]$} \\
\hline & Sensitivity to oxidative stress & iPSCs & $\uparrow$ & \\
\hline \multirow[t]{3}{*}{ In vivo } & MDA & Blood & $\uparrow$ & {$[41]$} \\
\hline & SOD activity & Blood & $\uparrow$ & \\
\hline & GPx & Blood & $\leftrightarrow$ & \\
\hline \multirow[t]{6}{*}{ Postmortem } & Lipid peroxidation & SN & $\uparrow$ & {$[38]$} \\
\hline & Reduced GSH & $\mathrm{SN}$ & $\downarrow$ & \\
\hline & Total iron & SN & $\uparrow$ & \\
\hline & Ferritin & SN & $\downarrow$ & \\
\hline & GPx activity & SN & $\downarrow$ & \\
\hline & Catalase activity & SN & $\downarrow$ & \\
\hline \multirow[t]{6}{*}{ Postmortem } & Iron & SN & $\uparrow$ & {$[39]$} \\
\hline & MAO-B & $\mathrm{SN}$ & $\uparrow$ & \\
\hline & $\mathrm{Cu} / \mathrm{Zn}-\mathrm{SOD}$ & SN & $\uparrow$ & \\
\hline & Heme oxygenase & SN & $\uparrow$ & \\
\hline & GSH & SN & $\downarrow$ & \\
\hline & Vitamin C & SN & $\downarrow$ & \\
\hline Postmortem & GPx 4 & SN & $\uparrow$ & {$[46]$} \\
\hline \multirow[t]{8}{*}{ Postmortem } & Protein oxidation & Caudate nucleus & $\uparrow$ & {$[35]$} \\
\hline & Lipid peroxidation & Frontal cortex & $\uparrow$ & \\
\hline & Protein nitration & $\begin{array}{l}\text { Caudate nucleus, putamen, } \\
\text { frontal cortex }\end{array}$ & $\leftrightarrow$ & \\
\hline & Total GSH & $\begin{array}{l}\text { Caudate nucleus, putamen, } \\
\text { frontal cortex }\end{array}$ & $\uparrow$ & \\
\hline & Catalase & $\begin{array}{l}\text { Caudate nucleus, putamen, } \\
\text { frontal cortex }\end{array}$ & $\leftrightarrow$ & \\
\hline & SOD & $\begin{array}{l}\text { Caudate nucleus, putamen, } \\
\text { frontal cortex }\end{array}$ & $\leftrightarrow$ & \\
\hline & GSH reductase & $\begin{array}{l}\text { Caudate nucleus, putamen, } \\
\text { frontal cortex }\end{array}$ & $\leftrightarrow$ & \\
\hline & GPx & Caudate nucleus, putamen & $\uparrow$ & \\
\hline
\end{tabular}

Abbreviations: 4-HNE: 4-hydroxynonenal, 8-OhdG: 8-hydroxydeoxyguanosine, CSF: cerebrospinal fluid, GPx: GSH peroxidase, GSH: glutathion, iPSCs: induced pluripotent stem cells, MDA: malondialdehyde, SN: substantia nigra, SOD: sodium dismutase, $\uparrow:$ increased, $\downarrow$ : decreased, $\leftrightarrow$ : not different. 
neurons, and this will expose the neurons to increasing concentrations of highly toxic hydroxyl radicals. Moreover, several studies have indicated that one of the consequences of normal aging is the increase in the concentrations of copper and iron in brain tissue [51]. Due to this, the current view is that the hydroxyl-radical-mediated oxidative stress induced by dysregulation in the neuromelanin and iron homeostasis is one of the most important contributors to the oxidative damage in $\operatorname{PD}[17,36,51]$. The generation of ROS also initiates excitotoxicity, which is modulated by the overactivation of N-methyl-D-aspartate (NMDA) receptors [51]. In response to activation of the NMDA receptors, NO is produced, due to the interaction of NOS with these receptors [54].

Numerous studies have reported the involvement of mitochondria, neuroinflammation via activated microglia, and other ROS-mediated pathways in the pathogenesis of PD.

3.1. Impaired Mitochondrial Function. Several lines of evidence, some obtained from PD patients, indicate that the mitochondrial dysfunction plays a major role in the pathogenesis of PD, with defects in complex I of the mitochondrial electron chain transport gaining the most attention $[9,57$, 58]. Complex I is located in the inner mitochondrial membrane and constitutes a part of the oxidative phosphorylation system responsible for the generation of cellular adenosine$5^{\prime}$-triphosphate (ATP) [59, 60]. Mitochondria exert both vital and lethal functions in physiological and pathological conditions. On the one hand, they are indispensable for energy production and hence for the survival of eukaryotic cells; on the other hand they are crucial regulators of the intrinsic pathway of apoptosis [61]. Furthermore, mitochondria are main sources of ROS in the central nervous system (CNS) [62]. Mitochondria contain redox carriers that can transfer single electrons to oxygen, thus generating $\mathrm{O}_{2}{ }^{\bullet-}$. Enzymes in the tricarboxylic acid cycle and the electron transport chain (complexes I, II and III) and MAOs are among the mitochondrial carriers generating $\mathrm{O}_{2}{ }^{\bullet-}$. The generated $\mathrm{O}_{2}{ }^{--}$can be converted by the cellular defense mechanism into $\mathrm{H}_{2} \mathrm{O}_{2}$, which further can react with other molecules, as described above [30,62].

An impairment of normal mitochondrial function leads to an excessive production of ROS and a general decrease in ATP levels. Furthermore, there is a concomitant loss of mitochondrial membrane potential [63]. Under physiological conditions, mitochondria harbour a robust mitochondrial transmembrane potential and a low-conductance state of the permeability transition pore complex (PTPC) might contribute to exchange of small metabolites between the cytosol and the mitochondrial matrix, a process that is mainly controlled by mitochondrial solute carrier. In response to proapoptotic stimuli, such as ROS and calcium $\left(\mathrm{Ca}^{2+}\right)$ overload, the PTPC assumes a high conductance state that allows the deregulated entry of small solutes into the mitochondrial matrix along their electrochemical gradient. This phenomenon, which is known as mitochondrial permeability transition, results in the immediate dissipation of the mitochondrial membrane potential and osmotic swelling of the mitochondrial matrix. As a consequence, this may lead to mitochondrial outer membrane permeabilization and to the release into the cytosol of cytotoxic proteins normally confined within the mitochondrial intermembrane space. The cytotoxic proteins include caspase activators, such as cytochrome $\mathrm{c}$ and DIABLO, as well as caspaseindependent cell death effectors like apoptosis-inducing factor and endonuclease $\mathrm{G}[61,63]$.

Next to being the main source of free radicals and having a role in the electron transport chain and oxidative phosphorylation, the mitochondria are also involved in $\mathrm{Ca}^{2+}$ homeostasis [9]. Mitochondria are known to play an important role as $\mathrm{Ca}^{2+}$ buffer, thereby preventing sustained high cytosolic $\mathrm{Ca}^{2+}$ levels, via the uptake of these ions through a membrane potential-dependent uniporter [64]. Saturation of this buffering system can be prevented, by releasing mitochondrial $\mathrm{Ca}^{2+}$ again into the cytosol via the $\mathrm{H}^{+} / \mathrm{Ca}^{2+}$ exchanger and a yet unidentified $\mathrm{Na}^{+} / \mathrm{Ca}^{2+}$ exchanger [65].

3.2. Neuroinflammation. The neurotoxicity associated with inflammatory processes in the CNS seems to be mainly mediated by overactive microglia [66]. Microglia are components of the CNS innate immune system, normally display a resting phenotype, and only become activated upon brain injury or immune challenge [66]. In relation to oxidative stress, microglia have been identified as an important source of ROS [62]. Microglia, which are phagocytic cells, have a dual role in the CNS. They can be either neuroprotective through the elimination of exogenous and endogenous substances or promote neurodegeneration by producing potentially toxic agents [66-68]. Overactive microglia were shown to release toxic compounds to dopaminergic cells, such as $\mathrm{O}_{2}{ }^{\bullet-}, \mathrm{H}_{2} \mathrm{O}_{2}$, and $\mathrm{NO}$, as well as proinflammatory cytokines, such as the tumor necrosis factor-alpha (TNF- $\alpha$ ) and interleukin- $1 \beta$ (IL$1 \beta)[69,70]$, and it is currently well known that microglial activation results in dopaminergic cell death in $\mathrm{PD}$ patients [71]. $\mathrm{O}_{2}{ }^{\bullet-}$, released by overactive microglia, seems to be the critical mediator of cell death [71]. Its production is mediated by the NADPH oxidases, which comprise several multicomponent enzyme complexes, that transfer electrons across biological membranes [62, 71-73]. Although the major source of NADPH oxidase induced oxidative stress is of activated microglia, NADPH oxidases from neuronal origin might also contribute to cell death [74]. The activation of NADPH oxidase is also mediated via interactions between the dopaminergic system and the central renin-angiotensin system [75]. We recently showed that stimulation of central angiotensin 1 (AT1) receptors leads to the activation of NADPH oxidase, while stimulation of central AT2 receptors leads to inhibition of NADPH oxidase activation $[75,76]$. Factors driving microglial overactivation in PD might be linked to environmental toxins, endogenous disease proteins, and even the neurodegenerative process itself. Indeed, it was shown that PD toxins such as MPTP, rotenone, paraquat, and particulate matter from polluted air are potent inducers of microglial activation, suggesting environmental triggers in $\mathrm{PD}[66]$. 
Lastly, dopaminergic cell death releases proteins in the extracellular space, such as histones, oxidized lipids, DNA, and ATP, that are interpreted by microglial cells as damageassociated molecular pattern molecules, leading to their activation [77]. This finding is particularly interesting, as it implies that neuronal cell death, which may be increased by microglial neurotoxicity, further reinforces the overactive phenotype of the microglia. This would create a selfperpetuating neurotoxic cycle, whereby microglial cells are capable of enhancing neurodegeneration, while this in turn would further enhance microglial activation [66]. Such a cycle would be particularly damaging to dopaminergic neurons in the $\mathrm{SN}$, as it contains 4.5 times more microglial cells compared to other areas of the brain [71].

Understanding the role of oxidative stress as a primary event in the pathogenesis of PD is complicated, as different pathways themselves can play a primary role and, in turn can initiate the formation of ROS/RNS. The above findings demonstrate how complex the interaction between oxidative stress, mitochondrial impairment, and neuroinflammation is, making it problematic to determine which occurs first. Furthermore, impairment in the UPS system, which is the primary mechanism responsible for eliminating mutant, damaged, and misfolded intracellular proteins, and for regulating the levels of short-lived proteins, can result in the accumulation of abnormal proteins, leading to the disruption of the cellular homeostasis and integrity, and creating a state referred to as proteolytic stress [78]. Again, oxidative stress may contribute to this impairment, and in turn, UPS impairment can induce oxidative stress [17].

3.3. Cellular Antioxidant Mechanisms. To maintain a proper redox balance, all aerobic organisms utilize series of sophisticated antioxidant defense mechanisms in an attempt to protect themselves against oxidative damage by eliminating ROS, thereby balancing the ratio between generation and detoxification of ROS. Numerous antioxidant enzymes, such as catalases, SOD, peroxiredoxins, and GSH peroxidase (GPx), and low-molecular weight compounds such $\alpha$ tocopherol, ascorbic acid, and GSH have been characterized $[31,37,79,80]$. The removal of $\mathrm{H}_{2} \mathrm{O}_{2}$ is accomplished by two enzyme families: GPx and catalases, both reducing it to oxygen and water. GPx utilizes the power of reduced GSH that is converted, via reduction of $\mathrm{H}_{2} \mathrm{O}_{2}$, to oxidized GSH [79]. The continuous action GPx is permitted by GSH reductase that recycles reduced GSH from its oxidized form. Another enzyme, quinone reductase, catalyzes the direct divalent reduction of quinones to quinols and avoids the formation of reactive semiquinone radical intermediates [79]. Furthermore, two nonenzymatic proteins, ferritin and ceruloplasmin, have an important role in transition metal storage [79].

In addition, a rise in ROS may also constitute a stress signal that activates redox-sensitive signaling pathways, which once activated may have either damaging or potentially protective functions [80]. The major signaling pathways activated in response to oxidative stress are the extracellular signal-regulated kinase (ERK), c-Jun $\mathrm{N}$-terminal kinase (JNK), phosphoinositide 3-kinase $[\mathrm{PI}(3) \mathrm{K}] /$ protein kinase
B (AKT), nuclear factor kappa B $(\mathrm{NF} \kappa \beta), \mathrm{p} 53$ and $\mathrm{p} 38$ pathways. NF $\kappa \beta$ and p53 are transcription factors, while $\mathrm{PI}(3) \mathrm{K} / \mathrm{AKT}$ and mitogen-activated protein kinase (MAPK) pathways regulate transcription factors through phosphorylation. The degree to which a given pathway is activated is highly dependent on the nature and on the duration of the stress [80]. Furthermore, the activation of peroxisome proliferator-activated receptors (PPAR), whose natural ligands are polyunsaturated fatty acids and their oxidation products, may be involved in the pathogenesis of PD. PPAR is also considered as a regulator of mitochondrial biogenesis genes that upregulates genes known to protect against oxidative stress [81]. Numerous protective genes, such as hemeoxygenase, have been shown to be inducible in mammalian cells following exposure to oxidative stress [79]. The exact ROS sensing mechanisms are not well understood, but a number of transcription factors that regulate the expression of antioxidant genes are well characterized [37]. Major signaling pathways activated in response to oxidative stress include (i) transcription factors, such as NFkb, activator protein 1 (AP1), and antioxidant response elements (AREs) binding proteins that can interact directly with specific DNA motifs on promotors of target genes (ii) or via MAPK cascades, which in turn activate transcription factors that trigger target gene transcription [82]. The transcription of the cytoprotective proteins is under control of the nuclear transcription factor NF-E2-related factor 2 (Nrf2). Upon normal homeostatic conditions, Nrf2 is repressed by its negative regulator Keap1. Upon exposure to ROS, Nrf2 dissociates from cytosolic Keap1 and translocates to the nucleus, where it binds to the AREs in the promoter region of the genes encoding antioxidant enzymes [37]. These findings suggest that an increase in ROS results in two critical effects: (i) damage to cell components via an excessive production of ROS and also (ii) activation of specific signaling pathways regulated via ROS, influencing various cellular processes leading either to cell death or to proper cell function $[82,83]$.

Mitochondria are highly dynamic organelles that can undergo repeated cycles of fusion and fission to regulate their shape and length, and to control the total number of mitochondria per cell. The fusion of two mitochondria serves as a protective mechanism by forming tubular networks, leading to the exchange of lipid membranes, mitochondrial DNA (mtDNA), and soluble metabolites to compensate for individual deficiencies, as well as to the dilution of toxic intermediates, such as ROS. On the other hand, mitochondrial fission allows division and sequestration of damaged parts of this organelle by autophagy [84]. Several levels of defense mechanisms have been shown in mitochondria to ensure their integrity. A first line of defense comprises molecular chaperones, which assist folding and assembly of mitochondrial proteins and prevent oxidative-stressmediated mitochondrial injury. A second protective mechanism involves the degradation of damaged proteins by mitochondrial proteases [85]. However, once the mitochondrial quality control is overwhelmed, a third pathway is activated and induces selective autophagic clearance of damaged mitochondria (mitophagy) [85-87]. 


\section{Evidence of Oxidative Stress in Genetic Animal Models of Parkinson's Disease}

A better understanding of the pathophysiology of PD is only possible with the development of reliable experimental models, which can mimic disease processes with good fidelity. Initially, PD was modeled by administration of neurotoxins, such as 6-hydroxydopamine (6-OHDA) and MPTP. However, the identification of PD-associated genes has led to the development of new genetic animal models of PD [88]. While many models have been created, to date no single model, either based on toxins or genetics, has been able to recreate all the key features of the disease [89]. Nevertheless, knockout (KO), knockdown (KD), knock-in, overexpression, and/or mutations in single genes provide a powerful tool to study the etiology of PD [89]. Thus far, several genetically engineered animal models of PD have been generated $[55,90]$. Transgenic systems are widely used to study the cellular and molecular basis of human neurodegenerative diseases. A wide variety of model organisms have been utilized, including bacteria (Escherichia coli), nematodes (Caenorhabditis elegans), arthropods (Drosophila melanogaster), fish (zebrafish, Danio rerio), and rodents (mouse, Mus musculus and rat, Rattus norvegicus), as well as nonhuman primates (rhesus monkey, Macaca mulatta). These transgenic systems have enormous value for understanding the pathophysiological basis of disorders and have, in some cases, been instrumental in the development of therapeutic approaches to treat these conditions [91]. Once a causative mutation has been identified, transgenic systems can be generated to model the human disease or aspects of the altered gene function. All the models can provide further confirmation of the genetic basis of the disease and contribute to the identification of cellular and molecular mechanisms responsible for the disease phenotype. The available transgenic models vary in terms of easiness of manipulation and phylogenetic relatedness to humans, but have all been useful for the study of neurodegenerative diseases [91].

Among the invertebrate systems, the arthropod fruit fly Drosophila melanogaster and the nematode worm Caenorhabditis elegans have been the most widely used. The advantage of using invertebrates is their rapid production and analysis of transgenic lines expressing variants of diseaseassociated proteins. A disadvantage is that they lack critical factors, such as immune function and myelination [91, 92]. Despite the notable advances that have occurred in the above-mentioned species, the mouse remains the most widely used system for modeling human neurodegenerative diseases, since it is closely related to humans and offers advantages such as the relatively low cost, short generation time, high-environmental control, and the possibility for genome manipulation that allow virtually any diseaseassociated genetic alteration in humans to be introduced into the mouse [91].

Since, as described above, there is a prominent role for oxidative stress in the pathogenesis of $\mathrm{PD}$, while deficiencies in products of several PD-associated genes, including SNCA, parkin, PINK1, and DJ-1, may be linked to increased oxidative stress and/or higher vulnerability to oxidative damage, an overview is given of several oxidative stress hallmarks that have been observed within these genetically modified animal models of PD, with an emphasis on the mouse models (Table 2).

4.1. DJ-1 Models. No significant differences were detected in protein nitration, nucleic acid oxidation, and lipid peroxidation in the SN of aged DJ-1 KO mice [95]. Similarly, protein oxidation was not altered in the whole brain of DJ-1 KO mice at different ages $[93,94]$. These findings demonstrate that DJ-1 KO does not lead to increased oxidative damage in basal conditions [93-95]. Nevertheless, several groups observed increased mitochondrial ROS levels in the SN as well as in the whole brain, of DJ-1 KO mice at different ages [94, 96]. Furthermore, decreased mitochondrial aconitase activity was described in young, but not in aged $\mathrm{KO}$ mice [94]. It is interesting to note that although Andres-Mateos et al. [94] observed a 2-fold increase in the mitochondrial $\mathrm{H}_{2} \mathrm{O}_{2}$ production in DJ-1 KO brains, the mice did not show any dopaminergic degeneration or mitochondrial damage. They did note, however, that compensatory mechanisms (upregulation of MnSOD and GPx levels and increase in GPx activity) have been activated to compensate for the elevated $\mathrm{H}_{2} \mathrm{O}_{2}$ in mitochondrial, but not in cytosolic fractions from aged mice. These compensatory increases in GPx levels and activity appear to compensate for the lack of DJ-1 because the cytosolic levels of reduced GSH and GSSG and the ratio GSSG/GSH in DJ-1 KO brains do not differ significantly compared with their respective WT littermates [94]. These results taken together suggest that the absence of DJ-1 leads to an increase in mitochondrial $\mathrm{H}_{2} \mathrm{O}_{2}$ and a compensatory increase in mitochondrial GPx activity in aged DJ-1 KO mice, suggesting that DJ-1 plays an important role in mitochondrial elimination of $\mathrm{H}_{2} \mathrm{O}_{2}$ [94]. Furthermore, the decreased mitochondrial aconitase activity (an enzyme which is highly sensitive to ROS), as a consequence of an increase of mitochondrial $\mathrm{H}_{2} \mathrm{O}_{2}$ production, was only detected in young DJ-1 KO mice, and not in aged mice that showed an increased expression in GPx [94].

Recently, Guzman et al. [96] have shown, using transgenic mice that expressed a redox-sensitive variant of green fluorescent protein targeted to the mitochondrial matrix, that the engagement of plasma membrane L-type $\mathrm{Ca}^{2+}$ channels during normal autonomous pacemaking created an oxidative stress that was specific to the nigral neurons, and that the oxidative stress engaged defenses that induced transient, mild mitochondrial depolarization or uncoupling. This demonstrates that in mature SNc dopaminergic neurons a basal mitochondrial oxidant stress is present, and that the oxidative stress is not a consequence of old age, pathology, or the experimental preparation, but rather one of a neuronal design that engages L-type $\mathrm{Ca}^{2+}$ channels during autonomous pacemaking. Furthermore, $\mathrm{SNc}$ dopaminergic neurons lacking DJ-1 were shown to have an elevation in mitochondrial oxidative stress, and additional experiments confirmed that this was not due to lowered expression of antioxidant enzymes, but rather to diminished levels of 


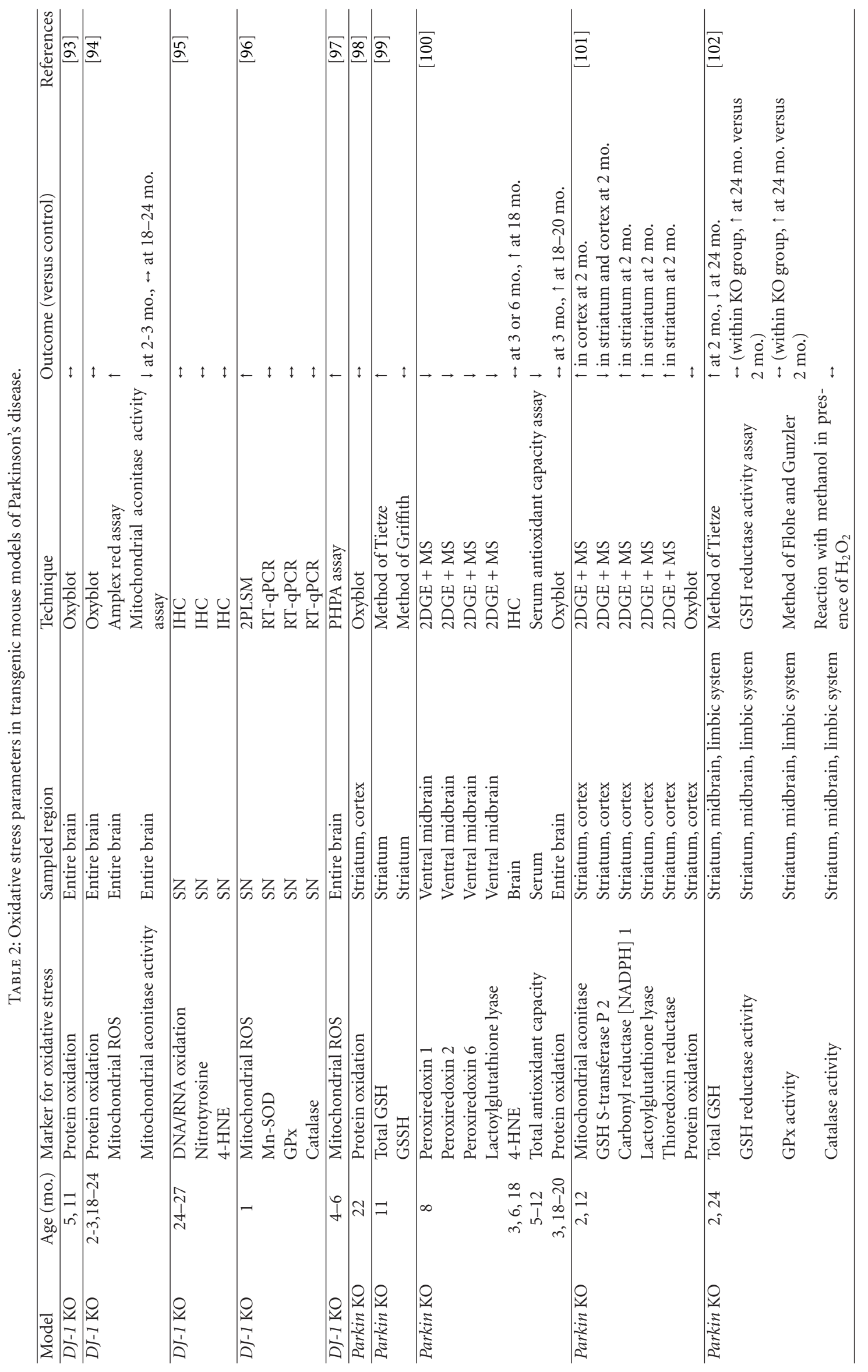




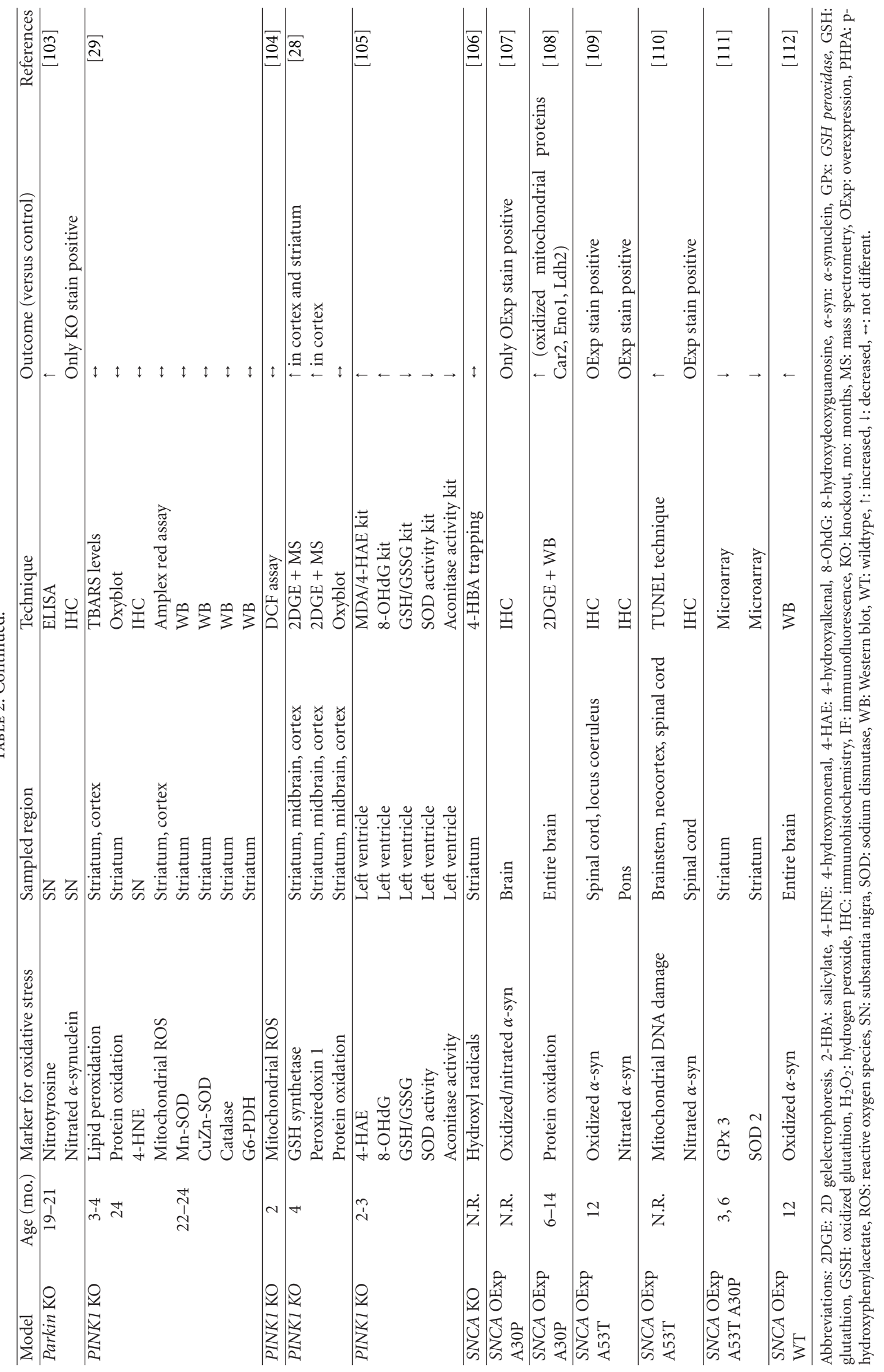


UCP4 and UCP5, comprising mitochondrial uncoupling in response to $\mathrm{Ca}^{2+}$-induced stress [96].

Furthermore, DJ-1 interacts with DAXX, a protein that binds to a fas death receptor and activates the JNK apoptotic pathway. By binding to DAXX, DJ-1 sequesters the protein into the nucleus and prevents it from gaining access to the cytoplasm and from inducing apoptosis at the fas death receptor. DAXX upregulation has been found to occur in cells that have been treated with $\mathrm{H}_{2} \mathrm{O}_{2}$, linking oxidative stress to this pathway. These data show that DJ-1 is involved in inactivating the apoptotic JNK pathway that is activated by oxidative stress, indicating its role in guarding against oxidative-stress-induced cell death [113].

4.2. Parkin Models. In line with the findings on DJ-1 deficient mice, no significant differences in protein oxidation have been found in different brain structures of parkin $\mathrm{KO}$ mice at the age of $2,3,12$, and 22 months $[100,101,114]$. However, when the entire brain was analyzed for protein oxidation, an increase was described in parkin $\mathrm{KO}$ mice at 18 to 20 months [100]. The same pattern was observed for lipid peroxidation in aged parkin KO mice [100].

Total striatal GSH levels were elevated in parkin $\mathrm{KO}$ mice at the age of 11 months, while no differences were observed in GSSG levels [99]. Rodríguez-Navarro et al. [102] also noticed increased total GSH levels in the striatum, midbrain, and limbic system of young parkin KO mice; however, total GSH levels were decreased at 24 months old of age in all the brain regions. No changes were observed in the activity of GSH reductase, catalase and GPx in the same brain regions when comparing age-matched parkin $\mathrm{KO}$, but an age-dependent increase was found for GPx and reductase within the parkin KO group [102].

It is interesting to note that the endogenous levels of DA were increased in the limbic regions of the above-mentioned mutant mice, while the DOPAC/DA and DOPAC/3methoxytyramine (3-MT) ratios were also increased. The increased DA levels and increased DA metabolism to DOPAC via $\mathrm{MAO}$, an enzyme considered to be mostly intraneuronal, and to 3-MT via COMT, an enzyme considered mostly extraneuronal, suggest that parkin dysfunction impairs the release of DA and increases intraneuronal DA metabolism via MAO. To determine in which cellular compartment DA is shifted to intraneuronal oxidation, the turnover of catecholamines after inhibition of their synthesis by $\alpha$-methyltyrosine $(\alpha-\mathrm{MT})$ was investigated. The rate of depletion of catecholamines in $\alpha$-MT-treated animals was similar in both mutant and WT animals, suggesting that the turnover is similar in both groups. Since the short-term effects of $\alpha$-MT on catecholamines are mostly related to the turnover of the newly synthesized intracytoplasmic pool of catecholamines, rather than the vesicular pool of catecholamines, these data suggest that the increased production of DOPAC in the absence of parkin is not mediated by changes in DA turnover, but due to intracytoplasmatic DA being unprotected against metabolism by MAO. Hence, monoamine metabolism in parkin mutant mice was shifted towards the $\mathrm{H}_{2} \mathrm{O}_{2}$ producing MAO pathway, with a compensatory increase in GSH levels.
Although there was no evidence for a reduction of nigrostriatal dopaminergic neurons in the parkin mutant mice, the level of DA transporter (DAT) protein was reduced in these animals, suggesting a decreased density of dopaminergic terminals or adaptative changes in the nigrostriatal system. Such an adaptive change was revealed by Itier et al. (2003) who noticed reduced amphetamine-induced DA release in neuronal cultures from parkin mutant mice compared to WT [99]. A similar decrease in evoked DA release in striatal slices was demonstrated by Kitada et al. [115]; however, the levels of total DA and striatal DAT were comparable between the experimental groups [115]. On the contrary, another study showed higher striatal levels of DA, DOPAC, and homovanillic acid (HVA) in parkin KO mice [116]. Similarly, increased DA levels in the midbrain, alongside a decrease in endogenous DA release after methamphetamine (METH) challenge shown by ex vivo autoradiography, and an upregulation of DA D1 and D2 receptor binding in the striatum were shown in parkin KO mice [117].

A proteomic analysis, performed by two-dimensional gel electrophoresis coupled with mass spectrometry, revealed several markers of oxidative stress to be upregulated or downregulated in either the ventral midbrain, or the striatum and cortex of parkin $\mathrm{KO}$ mice. In the ventral midbrain of 8 -month-old parkin $\mathrm{KO}$ mice, a decrease in peroxiredoxin 1, 2, 6 and lactoylglutathione lyase was observed [100]. Furthermore, a decreased antioxidant capacity was observed in the serum of parkin KO mice [100]. Several proteins involved in detoxification, stress-related chaperones, and components of the UPS were also altered in parkin KO mice [101]. These differences might reflect adaptive mechanisms aimed at compensating for the presence of ROS and the accumulation of damaged proteins in parkin $\mathrm{KO}$ mice. This study provides clues into possible compensatory mechanisms that protect dopaminergic neurons from death in parkin $\mathrm{KO}$ mice and may help to understand the preclinical deficits observed in parkin-related parkinsonism [101].

No gross mitochondrial abnormalities in striatal mitochondria from parkin $\mathrm{KO}$ mice were demonstrated, but reductions in the respiratory capacity were found [100]. Additional findings, which might be related to increased ROS, are an increased astrogliosis in the striatum and increased levels of microglial markers in the midbrain of aged parkin KO mice [102]. Furthermore, in aged parkin $\mathrm{KO}$ mice, higher levels of nitrotyrosine and nitrated $\alpha$-syn were found in the SN [103]. However, in another parkin KO model, immunohistochemical analysis of olfactory bulb, cortex, basal ganglia, midbrain, and brainstem of $\alpha$-syn, glial fibrillary acidic protein, and ubiquitin, as well as staining with thioflavin S, hematoxylin/eosin, and silver, revealed no differences between parkin null mice compared with WT mice $[114,118]$. Only one group demonstrated agedependent dopaminergic degeneration in the $\mathrm{SN}$ of parkin $\mathrm{KO}$ mutant determined by a significant loss of dopaminergic neuron terminals in the striatum and a reduction of striatal DA levels. Furthermore, age-dependent accumulation of proteinase-K-resistant endogenous $\alpha$-syn in the SN was found, which was colocalized with 3-nitrotyrosine (3-NT) [103]. However, earlier it was demonstrated that the ratio 
of prosurvival/proapoptotic proteins, as measured by the fraction $\mathrm{Bcl} 2 / \mathrm{Bax}$, is reduced in the striatum and the midbrain of young mature and of aged parkin $\mathrm{KO}$ mice with respect to their respective age-matched controls. This was associated with a greater number of apoptotic cells with fragmented DNA in the striatum and SN of 24-month-old parkin $\mathrm{KO}$ mice than in any other group [102].

4.3. PINK1 Models. Similarly, as with the other mouse models, no significant differences in protein oxidation and lipid peroxidation have been found in different brain structures of PINK1-deficient mice [28, 29]. However, in the left ventricle of PINK1 KO mice, increased lipid peroxidation and DNA oxidation were observed [105].

The role of PINK1 in the maintenance of basic functions of mitochondria was investigated by several groups [30, 119]. In this perspective, PINK1 KO mice did not reveal any changes in the ultrastructure or the total number of mitochondria $[29,120]$. Nevertheless, some of the functional aspects of the mitochondria were found altered in PINK1 $\mathrm{KO}$ mice. Reduced mitochondrial respiration was shown in a brain-region-specific manner in young PINK1 KO mice, with the impairment present in the striatum, which is rich in dopaminergic nerve terminals, but absent in the cerebral cortex, suggesting that high concentrations of DA in the striatum may render the striatum more sensitive to the loss of PINK1 than in the cerebral cortex [29]. Consistent with the presence of oxidative stress in the striatum, and independent of the genotype, an increased lipid peroxidation was present in the striatal mitochondria relative to cortical mitochondria [29]. Furthermore, in the striatum of PINK1 $\mathrm{KO}$ mice, no differences in different antioxidant proteins could be observed [27, 29].

However, with a large proteomic screening, increased levels of GSH synthetase in the striatum and cortex and increased levels of peroxiredoxin 1 in the cortex were observed in PINK1 KO mice [28]. Within the left ventricle of PINK1 KO mice, decreased activities of SOD, and of mitochondrial and cytoplasmic aconitase were observed, associated with a decreased ratio GSH/GSSG [105].

Mitochondria isolated from PINK1 KO mice had significantly reduced membrane potential [55]. As has been noted previously, under conditions of oxidative stress, the cysteines of the mitochondrial permeability transition pore undergo oxidation, thus increasing the probability for the pore to open and collapse the transmembrane potential. In conditions of PINK1 deficiency, the changes in mitochondrial transmembrane potential are independent of the mitochondrial permeability transition pore regulation, suggesting that other pathways are involved [29]. PINK1 KO mice do not show any loss of dopaminergic neurons and have normal levels of striatal DA and DA receptors [88, 121]. Although silencing of PINK1 does not cause degeneration, it induces some functional deficits, such as a decrease in evoked extracellular striatal DA levels, which may lead to a decreased activation of postsynaptic DA 1 and DA 2 receptors and, as a consequence, to defects in striatal plasticity and in spontaneous locomotor activity $[121,122]$. It has also been observed that silencing of PINK1 expression in mice using RNA interference did not change the striatal DA content and the nigral dopaminergic neuron number [123]. These observations strengthen the hypothesis that PINK1 has a critical role in DA release and that loss of PINK1 may play a role in mechanisms preceeding nigrostriatal degeneration.

It has been demonstrated that PINK1 can protect mitochondria via the activation of mitochondrial chaperones TRAP-1 and HtrA2. In vitro studies lacking these chaperones, such as the study by Gautier et al. [29], only support a direct protective role of PINK1 [29]. The neuroprotective mechanisms of PINK1 might involve the phosphorylation of these key signaling proteins in pathways governing cell death and survival. Upon phosphorylation, these proteins may mediate neuroprotective signals of PINK1 by different mechanisms. Upon phosphorylation, TRAP-1 is activated and blocks generation of mitochondrial ROS. HtrA2 phosphorylation protects neuronal cells from undergoing apoptosis induced by neurotoxins and oxidative stress [124].

Several studies in PINK1-deficient neuronal cells and fibroblasts from patients carrying PINK1 mutations also revealed increased levels of mitochondrial ROS and lipid peroxidation [125]. The oxidative stress observed in these patients or in PINK1-deficient cells can also result from reduced antioxidant defense mechanisms. Indeed, Gegg et al. [126] demonstrated that silencing PINK1 resulted in decreased cellular levels of reduced GSH, as reduced GSH levels were decreased in human PINK1 KD cells, as well as in human fibroblasts derived from PINK1 patients [126]. Mitochondrial $\mathrm{Ca}^{2+}$ overload and a concomitant impaired buffering capacity were also observed due to PINK1 deficiency and appeared to be caused by a defective $\mathrm{Na}^{+} / \mathrm{Ca}^{2+}$ antiporter function $[27,65]$. An indirect role for PINK1 in $\mathrm{Ca}^{2+}$ signaling in zebrafish has been proposed [64]. Using a yeast two-hybrid screen, the interaction of PINK1 and a $\mathrm{Ca}^{2+}$ sensing molecule, neuronal $\mathrm{Ca}^{2+}$ sensor 1 , has been demonstrated [64]. This sensor may be involved in the regulation of DA 2 receptor expression [127] and the release of neurotransmitters [128]. An increased $\mathrm{Ca}^{2+}$ induced mitochondrial permeability transition was shown in purified brain mitochondria of PINK1 KO mice [29]. Accumulation of phospho-c-jun in neurons of the SN of PINK1 KO mice was shown, suggesting that JNK activity in PINK1 KO mice is increased. Therefore, the increased mitochondrial $\mathrm{Ca}^{2+}$ sensitivity and JNK activity are early defects in PINK1 KO mice that precede reduced DA levels and abnormal DA homeostasis, and that may contribute to neuronal dysfunction. Differential gene expression in the nigrostriatal system of PINK1 KO mice supports early dopaminergic dysfunction and shows that PINK1 deletion causes aberrant expression of genes that regulate innate immune responses [104]. Striatal gene expression profiles between 2-month-old WT and PINK1 KO mice have been performed. Several of the upregulated genes encoded stressinducible transcription factors of the activating transcription factor (ATF,) and AP1 families, including ATF-3, cfos, FosB, JunB and Egr-2. ATF3 is induced by multiple signals, including inflammatory cytokines, DNA-damaging agents, and physiological stressors. Interestingly, increased 
striatal expression of Fos-related antigens and JunB has been observed following neuronal injury and degeneration in the DA system. Moreover, striatal c-fos expression is regulated by DA and, in the DA-depleted striatum, may be induced via compensatory supersensitivity of DA receptors [104].

Apart from complex I inhibition, recent genetic data point to PD-associated genes that are linked with normal mitochondrial function and resistance against oxidative stress, such as parkin, PINK1, and DJ-1. All these genes are believed to play an important protective role within the mitochondria by preventing mitochondrial dysfunction mediated by oxidative stress [125]. This protective role might be mediated by parkin's ubiquitin ligase function. Indeed, production of ROS is a physiological consequence of the mitochondrial respiratory chain, and parkin might play a role in removing oxidatively damaged proteins that can impair normal mitochondrial function. However, it is equally possible that parkin's protective action may be mediated via its signalling function [24]. DJ-1 can be considered as a redox-active molecule that can sense oxidative stress via oxidation of an internal cysteine residue, followed by translocation to the mitochondrial matrix and intermembrane space [9]. Once localized to the mitochondria, DJ-1 can act as a molecular chaperone to protect against oxidative stress [129]. Furthermore, DJ-1 localized in the intermembrane space can act as a free radical scavenger, preventing the accumulation of free radicals derived from the mitochondrial respiratory chain [63].

Interestingly, recent insights indicate the collaboration of all mitochondrial PD genes, DJ-1, PINK1, and parkin, in protecting the mitochondria against oxidative stress [7]. Furthermore, parkin and PINK1 seem to act in concert to mediate the autophagic degradation of terminally damaged mitochondria, functioning as a global mitochondrial quality control mechanism $[119,130,131]$. Such a quality control mechanism is important to the cell, as damaged mitochondria are sensitive to rupture and release of proapoptotic mediators via a selective form of macroautophagy known as mitophagy [130], and loss of this protective function may be an important cause of neurodegeneration in PD, as neuronal death in PD appears to occur by apoptosis rather than necrosis [17]. Consistently parkin can partially compensate for PINK1 loss when being overexpressed [132]. Nevertheless, a recent study demonstrated that even the inactivation of the genes PINK1, DJ-1, and parkin is insufficient to cause significant nigral degeneration within the lifespan of the mice, suggesting that these genes may be protective to external stressors rather than being essential for the survival of dopaminergic neurons during the normal aging process [133].

4.4. SNCA Models. Under physiological conditions, there was no difference in the basal hydroxyl radical production in the striata of WT and homozygous SNCA KO mice, suggesting that $\alpha$-syn deficiency does not affect basal hydroxyl radical production [106]. As has been noted previously, $\alpha$ syn is a specific target of nitration in the SNc of PD patients, suggesting the role of oxidative damage in the formation of $\alpha$-syn inclusions [134]. Similarly, an increased oxidative damage, as shown by the presence of oxidized and nitrated $\alpha$-syn, was shown in mice that overexpress mutant or WT $\alpha$ syn $[107,109,110,112]$. In addition, in the A30P mutant mouse model, increased levels of oxidized mitochondrial proteins were found [108], whereas in mice overexpressing the mutant A53T form of $\alpha$-syn, increased mtDNA damage was demonstrated [110]. The presence of mtDNA damage is possibly related to oxidative stress, since the presence of nitrated $\alpha$-syn was shown in the same mouse model [110].

Moreover, in double mutant $\alpha$-syn transgenic mice, decreased expression levels of GPx 3 and SOD-2 were described, yet this still needs to be confirmed at the protein level [111]. Furthermore, an A53T $\alpha$-syn transgenic mouse model was found to have higher DJ-1 levels at 3 months of age, suggesting an attempt to preserve mitochondrial function and reduce oxidative burden [55]. Furthermore, it was shown that $\alpha$-syn is colocalized with the JNK interacting protein (JIP). This proposes a protective role for $\alpha$-syn against oxidative stress, due to its ability to inactivate the JNK pathway via upregulation of JIP. The protective activity of $\alpha$-syn may be decreased when the protein is mutated, misfolded, or aggregated and therefore unable to interact with JIP [113]. Growing evidence indicates a connection between $\alpha$-syn, oxidative stress, and mitochondrial dysfunction in PD $[54,81]$. In this connection, $\alpha$-syn can bind to mitochondrial complex I and inhibit its activity, illustrating one way in which excess $\alpha$-syn can aberrantly induce mitochondrial dysfunction [55]. The oxidative events seem to be fairly target specific, concerning the modified amino-acid residue. Indeed, for instance, the nitration of tyrosine residues within the $\alpha$-syn protein is found to accumulate in the LBs [36].

The main involvement of SNCA in PD is via $\alpha$-syn aggregation, as $\alpha$-syn oligomers are considered as an important source of cell toxicity for dopaminergic neurons [129]. Selective damage to both soluble and insoluble $\alpha$-syn, which increases the tendency to aggregate, has been demonstrated in the brains of PD patients [81]. Free radicals may lead to the stabilization of a toxic protofibril of $\alpha$-syn, and this stabilization appears to depend on the oxidative ligation of $\alpha$-syn to DA [81]. Furthermore, $\alpha$-syn is involved in synaptic vesicle homeostasis and the storage of DA in synaptic vesicles, and the loss of normal $\alpha$-syn function following aggregation can lead to increased levels of oxidative stress [56]. Furthermore, the described mutations reduce the affinity of $\alpha$-syn for lipids, therefore increasing the cytoplasmic pools of the protein, and this in turn can be affected by several mechanisms [23]. Furthermore, it is known that lipid-bound WT $\alpha$-syn is a potent inhibitor of phospholipase $\mathrm{D} 2$, an enzyme that hydrolyzes phospholipids, and that is involved in synaptic vesicle recycling [56]. As a consequence of the mutations, a decreased phospholipase D2 inhibition will in turn lead to enhanced and dysregulated synaptic vesicle recycling and reduced synaptic vesicle formation. The inability of the neurons to from synaptic vesicles from the early endosome may result in a decreased number of vesicles available for DA storage, leading to accumulation of DA in the cytoplasm [56]. It was shown that toxic oligomeric aggregates of $\alpha$-syn can also bind to and form pores in 
the vesicle membrane, thereby releasing the remaining DA vesicular stores in the cytoplasm [63]. As a consequence, mutations in SNCA can lead to both loss of normal $\alpha$-syn function in synaptic vesicle dynamics, and gain-of function of toxic $\alpha$-syn aggregation [56], both pathways leading to a higher content of cytoplasmic DA, which in turn considerably increases cellular oxidative stress [23]. Mutations in SCNA have also been shown to have a modulatory action on tyrosine hydroxylase (TH) activation, inhibit of vesicular monoamine transporter 2 activity, interact with septin4 [135], and alter the expression of dihydropteridine reductase [51]. Furthermore, a complex formation with the human DAT has been shown, thereby inhibiting the uptake of DA by its transporter [51].

Recently, induced pluripotent stem-cell-derived dopaminergic neurons obtained from a PD patient with SNCA triplication showed increased levels of oxidative stress and were more sensitive to $\mathrm{H}_{2} \mathrm{O}_{2}$-induced oxidative stress [45]. Additionally, $\alpha$-syn levels increase within the dopaminergic neurons with aging, an important etiological factor of PD [24]. These findings and the intrinsic potential for the oxidative metabolism of DA to generate ROS have suggested that oxidative stress may be involved in the degeneration of the dopaminergic neurons in the SNc of PD patients [134].

Altogether, these findings indicate that genetic alterations in the PD genes parkin, PINK1, DJ-1, and SNCA can contribute to enhanced levels of oxidative stress. Even though the precise mechanistic subtleties are yet to be revealed, the working hypothesis based on the previously presented data is that they could enhance cellular oxidative stress by contributing to oxidative stress generating pathways in dopaminergic neurons, such as improper DA handling, mitochondrial dysfunction, and neuroinflammation (Figure 1).

\section{Gene-Environment Interactions in Mouse Models of Parkinson's Disease}

Besides the genetic background, environmental factors, such as environmental toxins or lifestyle factors, may have a role in $\mathrm{PD}$ pathogenesis [24].

5.1. Environmental Toxins. Hints that environmental toxins might play a role in the molecular pathology of PD first appeared after the accidental administration of MPTP in a group of young intravenous drug users, who eventually developed a clinical phenotype reminiscent of late-stage PD, albeit in the absence of LB pathology [69]. It was the first proof that the exposure to an environmental toxin could produce parkinsonism in humans. MPTP was subsequently identified as a potent neurotoxin that can easily cross the blood-brain barrier, being metabolized to 4-phenyl2,3-dihydropyridinium ion $\left(\mathrm{MPP}^{+}\right)$in astrocytes, a potent mitochondrial complex I inhibitor, that is then selectively transported into dopaminergic neurons via DAT, ultimately leading to cell death via mitochondrial impairment [24]. In addition to MPTP, other environmental toxins, such as the herbicide paraquat and rotenone have been identified as contributors to dopaminergic neuronal cell death and parkinsonism, supporting further the link between environmental exposure to pesticides and a risk of developing PD. Interestingly, a recent meta-analysis of 19 studies on the involvement of environmental pesticides in the pathogenesis of PD found an estimated doubling of the risk to develop PD [7]. Four individual pesticides were found to increase the risk of PD: dieldrin, maneb, paraquat, and rotenone [24], the latter two behaving as mitochondrial toxins, in a similar manner to MPTP [7].

5.2. Lifestyle Factors. Lifestyle factors and dietary habits have also been shown to influence the risk of developing PD. Epidemiological data showing that consumption of coffee, tobacco, and nonsteroidal anti-inflammatory drugs reduces the risk of PD are intriguing $[1,55]$. A high lipid consumption and caloric intake have been investigated as potential risk factors in PD. This was confirmed by a study showing that eating foods high in animal fat is associated with increased risk of PD [136].

5.3. Gene-Environment Interactions. As previously noted, the current view is that the ethiopathogenesis of PD is fueled by an interplay of genetic, environmental, and age-related factors, with gene-environment interactions playing a critical role [24]. In certain cases, genetic susceptibility is insufficient to lead on its own to PD [24]. For instance, the penetrance of some monogenic forms of $\mathrm{PD}$ is incomplete and variable, such as the penetrance of the Gly2019Ser mutation in LRRK2 [24]. Therefore, other factors, such as age and environmental exposure to certain toxins, might contribute to the pathology. At the same time, the converse is also true, as the risk of developing PD after toxin exposure also seems to be determined in part by genetic factors. Indeed, it seems that the presence of mutations that predisposes to spontaneous neurodegeneration are able to increase the endogenous toxicity of environmental toxins [24]. Recent studies also suggest that environmental factors may contribute to neurodegeneration through the induction of epigenetic modifications, such as DNA methylation and chromatin remodeling, which may induce alterations in gene expression [137]. In PD, a direct relation between epigenetics and neurodegeneration has not been widely exploited yet [137]. It has been shown that methylation of the $\alpha$-syn intron 1 was reduced in DNA from $\mathrm{SN}$, putamen, and cortex in patients with sporadic PD. Methylation of this intron region regulates the $\alpha$-syn expression in experiments using a luciferase reporter, suggesting that hypomethylation of $\alpha$-syn intron 1 contributes to increased expression of $\alpha$-syn in PD. In turn the toxicity induced by $\alpha$-syn can be rescued by administration of histone deacetylase (HDAC) inhibitors in both cell culture and a transgenic fruit fly Drosophila model. Furthermore, HDAC inhibitors may be neuroprotective in dopaminergic neurons and in rodent models of PD [138]. Epigenetics may constitute the missing link in the interplay between genes and environment, despite this, it still remains largely unexplored [137]. 


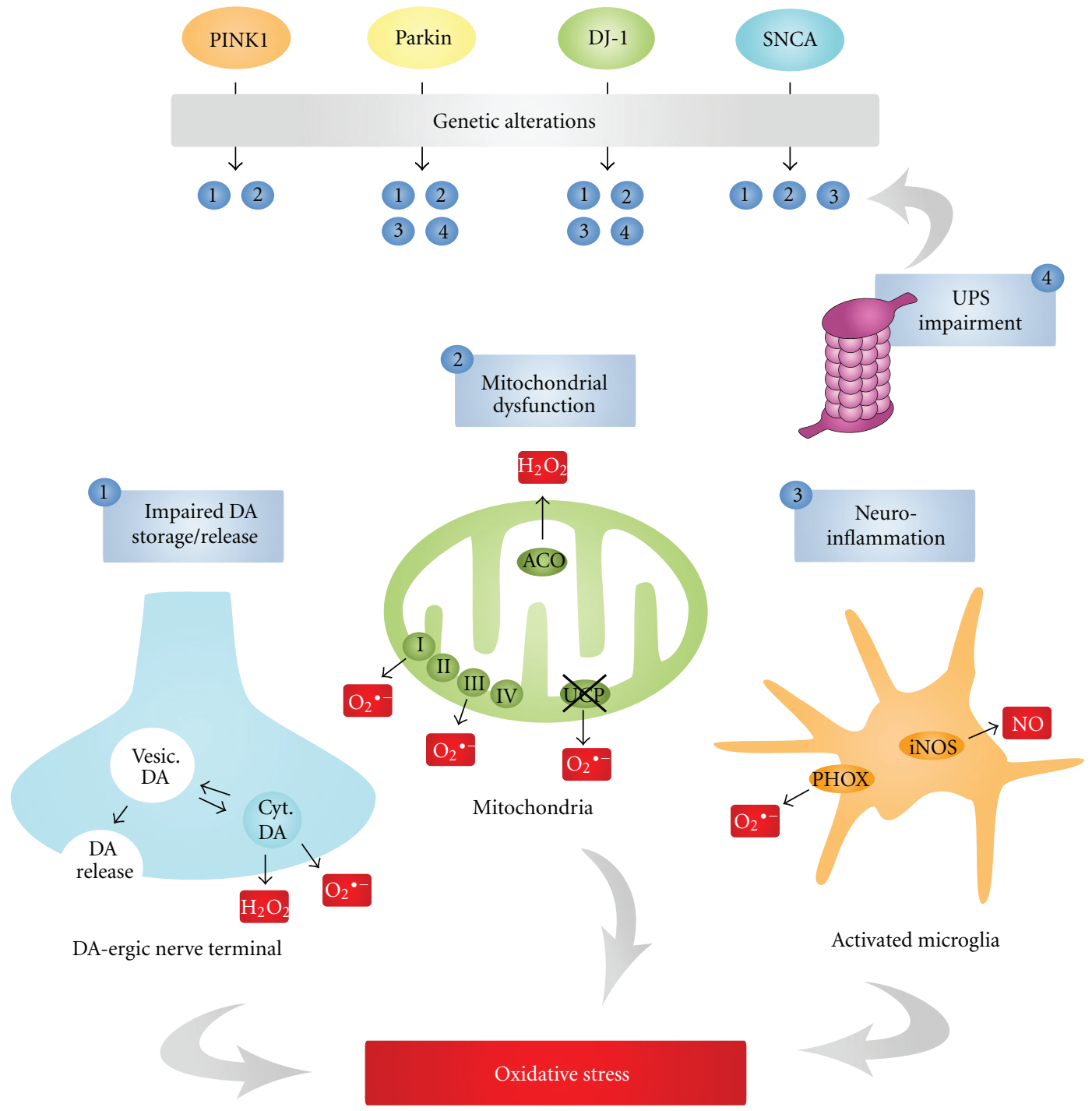

FIGURe 1: Pathways leading to oxidative stress in PD and the modulation by PD-related genes. Different pathways contribute to high levels of oxidative stress in dopaminergic neurons, including impaired DA handling (1), mitochondrial dysfunction (2), and neuroinflammation (3). As further discussed in the text, alterations in PD genes can potentiate all these impairments and, therefore, lead to enhanced levels of oxidative stress (see Table 2). UPS dysfunction (4), another important pathogenic pathway in PD, can contribute to the damaging effects of $\alpha$-syn, due to improper degradation of $\alpha$-syn mutants or oligomers. Abbreviations: ACO: aconitase, CYT: cytosolic, DA: dopamine, iNOS: inducible nitric oxide synthase, PHOX: NADPH oxidase, UPS: ubiquitin proteasome system, UCP: uncoupling proteins, VESIC: vesicular.

5.4. Toxin-Induced Models. The classical animal models of $\mathrm{PD}$ are based on the use of neurotoxins that reproduce some of the pathological and behavioural changes of the disease by inducing selective degeneration of the nigrostriatal system. The prototypical toxin-induced PD model is the 6-OHDA model, which is still the most commonly used procedure for obtaining PD-like loss of dopaminergic neurons in the SNc [139]. Until now, the neurotoxicity of 6-OHDA has been linked to (auto-) oxidation and the formation of quinones, $\mathrm{H}_{2} \mathrm{O}_{2}$ and oxygen radicals, intra- and extracellularly, and to inhibition of complex I and complex IV of the respiratory chain. Next to this, it has been shown to induce a massive release of DA in the extracellular space of the striatum, which in turn may contribute to oxidative stress. An increased level of DA may be correlated with an increased DA metabolism, which in turn may lead to an enhanced basal production of $\mathrm{H}_{2} \mathrm{O}_{2}$ and a depletion of GSH. It has also been demonstrated that nigrostriatal infusion of 6-OHDA is associated with an increased inflammatory response, due to activated microglia, which produce and release a broad spectrum of free radicals and inflammatory cytokines [139].

5.5. Genetic Models and Environmental Challenge. To complete our understanding of the genetic contribution to PD, a full characterization of the genetic architecture of the disease is needed, together with an exploration to the complex interaction between genetic and environmental factors. As the 
current genetic models of PD do not reproduce the complete disease pattern, the influence of genes, environment, and the interaction between both on different aspects of PD should be explored through the use of appropriate animal models. Nevertheless, genetic models can still provide interesting insights into the role of different genes in the pathogenic pathways of PD.

Integrating the use of neurotoxins, thereby simulating the environmental etiology, and the genetically engineered mice models, enables to simulate the gene-environment interactions that might occur during the development of PD. Consequently, a literature search was performed for studies where the susceptibility of genetic PD models to environmental stressors has been investigated (Table 3). Of course, not all environmental stressors used in the experimental settings are widely available to human exposure but should be considered as surrogate environmental stressors, as there is considerable overlap in pathogenic pathways. For example, 6-OHDA can be used as a surrogate stressor, as it has a similar mechanism of action as the more common environmental toxins, such as rotenone and paraquat. Furthermore, to increase the predictive validity of the animal model, the main etiological risk factor of $\mathrm{PD}$, namely, ageing, can be integrated within the model, thereby representing more realistic conditions.

\section{Transgenic Models in Conjunction with a Neurotoxin}

6.1. DJ-1 Mouse Models. DJ-1 KO mice of 2 to 4 months old were found to be significantly more susceptible to nigrostriatal deficits following systemic MPTP injection $[140,141]$. Furthermore, in WT mice, adenoviral-mediated overexpression of DJ-1 blocks MPTP-induced neuronal loss and protects the animals against neurodegeneration in the SNc $[140,142]$. Consequently, these findings point to a physiological role for DJ-1 in the protection of neurons against oxidative stress and environmental neurotoxins [140].

However, exposure of MPTP to differently generated DJ-1 KO mice resulted in striatal DA depletion with no differences in dopaminergic nigral cell loss [141]. Furthermore, enhanced DAT levels in mice deficient in DJ-1 protein were revealed when the transporter was evaluated in cell preparations enriched for synaptosomal membranes [141]. The discrepancy with the findings of Kim et al. [140] is likely due to differences among the various lines of DJ-1-deficient mice that differ in their sensitivity to MPTP $[140,141]$. One explanation could be that the C57BL/6 background strain of the DJ-1 KO mice confers higher sensitivity to MPTP than the B6/129 background of transgenic animals of ManningBog et al. [140, 141]. Indeed, since a couple of years, several reasons to avoid the use of MPTP have been proposed, including its strain-, age-, and gender-dependency in mice [150, 160-164].

6.2. Parkin Mouse Models. Parkin KO mice of 3 months of age challenged with the neurotoxins METH or 6-OHDA are not more sensitive compared to WT mice, as demonstrated by analysis of striatal DA levels [98]. METH is a toxin that releases vesicular DA into the cytoplasm and extracellular space and can inhibit mitochondrial function [98].

These findings suggest that the absence of a robust parkinsonian phenotype in parkin deficient mice is not due to the lack of exposure to environmental triggers with mechanisms of action similar to METH or 6-OHDA. Nevertheless, parkin-deficient mice could be more sensitive to other neurotoxins, such as rotenone or MPTP, which have different mechanisms of action [98]. Indeed, overexpression of parkin in combination with MPTP exposure appears to provide some protective benefits [142], whereas parkin delivery rescues $\alpha$-synucleinopathy in a rat model $[89,142]$.

6.3. PINK1 Mouse Models. The exposure of mitochondria isolated from the striatum or the cerebral cortex of aged PINK1 KO mice to mitochondrial toxins, such as paraquat, 6-OHDA, DA, and rotenone, does not lead to a different level of $\mathrm{H}_{2} \mathrm{O}_{2}$ production as in basal conditions [29]. PINK1 KO mice challenged with a systemic, acute lipopolysaccharide (LPS) injection and killed 8 hours after LPS administration demonstrated higher striatal expression levels of IL- $1 \beta$, IL12 , and IL-10. Striatal levels of 12 cytokines were not different versus WT without LPS challenge. Furthermore, a tendency for increased striatal expression of IL-2, IL-4, and TNF- $\alpha$ was observed after the LPS challenge. No differences were found in striatal CD3 expression before and after the LPS challenge. Isolated and cultured neonatal microglia from forebrain of PINK1 KO mice were also challenged with LPS. Higher expression of IL-10 and similar expression of IL-6, TNF$\alpha$, and granulocyte-colony stimulating factor (G-CSF) were found in cultured microglia after an LPS challenge [104]. In a recently published study, a conditional PINK1 RNA interference (RNAi) transgenic mouse model was exposed to paraquat. Mice were exposed to paraquat at the age of 14 days and further at the age of 12 weeks for 3 consecutive weeks. One week after the last paraquat injection, RNAimediated PINK1 KD was activated by injection with 4hydroxytamoxifen to induce Cre activity. At the age of 20 months, significant loss of dopaminergic neurons and loss of striatal DA was found in these mice [143]. Consistently, locoregional overexpression of PINK1 protects dopaminergic neurons from degeneration caused by MPTP [144]. Taken together, these data reveal an important in vivo role of PINK1 in protecting dopaminergic neurons against toxin-induced cell death.

6.4. SNCA Mouse Models. As shown by different groups, $\alpha$-syn-deleted mice are more resistant to MPTP neurotoxicity than their WT littermates, as demonstrated via striatal DA content determination and/or staining of the nerve terminals and/or dopaminergic nigral neurons [106, 145-149]. However, the intrastriatal administration of 3nitropropionic acid resulted in a significant increase in the striatal hydroxyl radical production in the WT mice, which was significantly attenuated in heterozygous and homozygous SCNA KO mice in a dose-dependent manner, suggesting that $\alpha$-syn deficiency results in neuroprotection 


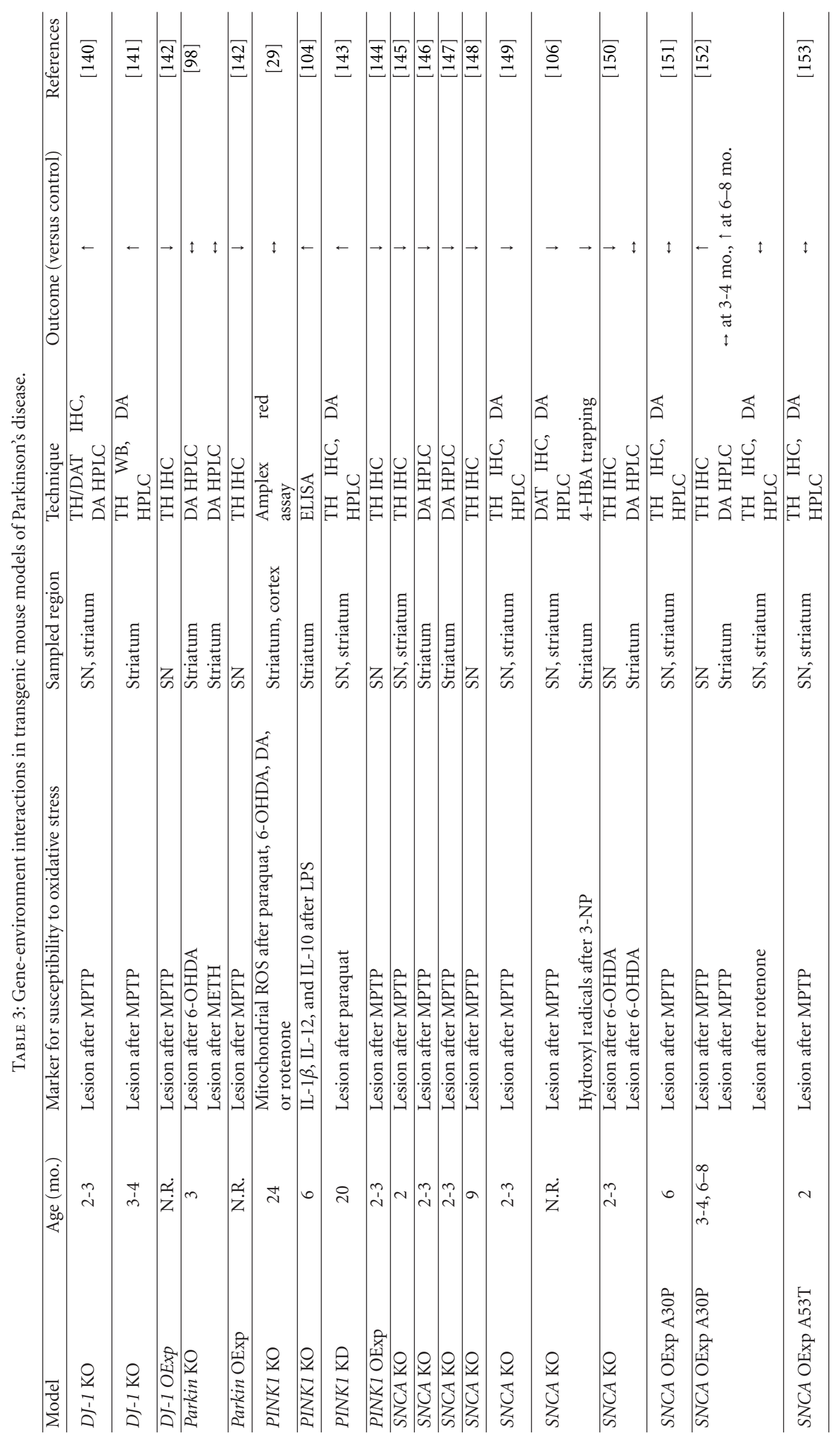




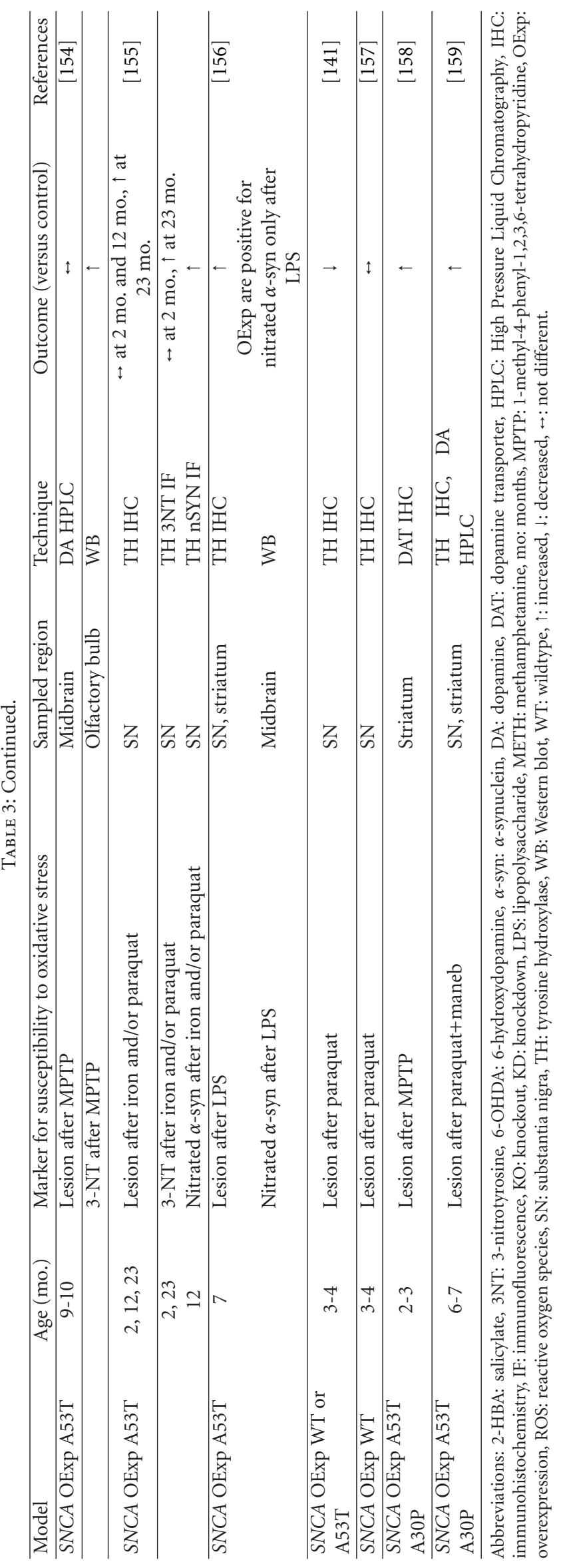


by reducing oxidative stress [106]. Consequently, Klivenyi et al. [106] concluded that the protective effects of $\alpha$-syn deletion were due to reduced hydroxyl radical formation [106]. One drawback of using 3-nitropropionic acid is its nonselectivity for dopaminergic neurons. Alvarez-Fischer et al. [150] checked the susceptibility of SNCA KO mice to 6OHDA and found that deletion of $\alpha$-syn rendered the mice more resistant to 6-OHDA and suggested that the toxicity of 6-OHDA was at least partially mediated by $\alpha$-syn [150]. These findings demonstrate that deletion of $\alpha$-syn results in neuroprotection, which might be mediated via inhibition of ROS formation [106, 150, 165, 166]. Another hypothesis suggests that aggregated, misfolded, and oxidized $\alpha$-syn, released or secreted from dying dopaminergic neurons, induces ROS formation, providing another mechanism by which ROS production could be attenuated in $\alpha$-syn-deleted mice [150]. Mice overexpressing the mutant $\alpha$-syn A30P under the control of a neuron-specific Thy-1 or a TH promoter are not more susceptible to MPTP compared to their WT littermates at the age of 6 months old [151]. These findings are in contrast with the increased susceptibility to MPTP of $\alpha$-syn A30P Tg5093 transgenic mice at 6-8 months of age. Furthermore, in the same mice, the lesion degree was not different from their nontransgenic littermate controls at the age of 3-4 months and a chronic rotenone treatment did not result in a different lesion degree [152].

Mice overexpressing unilaterally the mutant human $\alpha$ syn A53T in the SN are not more vulnerable to MPTP when compared to control [153]. The authors argue from their results against a direct function of (mutant) $\alpha$-syn in oxidative stress or apoptotic pathways [153]. On the contrary, transgenic mice expressing human A53T $\alpha$-syn are more sensitive to the neurotoxicity of MPTP when compared to non-transgenic littermates. Furthermore, MPTP treatment of the A53T transgenic mice was associated with a marked elevation in the 3-NT levels, which colocalized with $\alpha$-syn [154].

In another study by Peng et al. [155], the combined effects of neonatal iron feeding and environmental paraquat exposure on age-related nigrostriatal degeneration in transgenic mice expressing the A53T familial mutant form of human $\alpha$-syn was assessed in three age groups, namely, 2, 12, and 23 months old. Paraquat exposure reduced dopaminergic neurons in all three age groups to the same extent in WT and A53T $\alpha$-syn expressing transgenic mice, suggesting that paraquat toxicity is age-independent [155]. However, by 23 months of age, paraquat induced loss in dopaminergic cell numbers was more pronounced in those animals that had previously received elevated oral iron during the neonatal period, demonstrating that older iron-fed animals are more susceptible to exposure to the paraquat than younger ironfed animals and that paraquat administration accelerates nigral dopaminergic neuronal loss in these animals as a consequence of early iron exposure [155]. Furthermore, the neurodegeneration could be attenuated by a systemic treatment with the bioavailable antioxidant compound EUK-189. In addition, EUK-189 administration appeared to reduce the amount of colocalization of nitrated $\alpha$-syn within the nigral dopaminergic neurons, suggesting a preeminent role for oxidative stress in neurodegeneration and impairment of dopaminergic function in an experimental model of $\mathrm{PD}$, which combines environmental exposures with genetic factors known to separately be involved in the disease [155]. On the contrary, paraquat neurotoxicity assessed in mice overexpressing $\alpha$-syn, either the human WT or the A53T mutant form of the protein, displayed paraquatinduced protein aggregates, but were completely protected against neurodegeneration. Furthermore, increased levels of heat shock protein 70 were found, which could, therefore, contribute to neuroprotection in the transgenic mice [141].

In young and old double mutant (A53T and A30P) transgenic mice, the vulnerability against MPTP [158] and paraquat plus the fungicide maneb [159] was assessed. The enhanced toxicity of MPTP in the young double mutant mice could be due to a higher density of the DAT [158]. Double mutant mice expressing human A53T and A30P mutant forms of $\alpha$-syn were more sensitive to paraquat and manebinduced toxicity, when compared to mice expressing human WT alone [159].

In another study, 7-month-old transgenic mice expressing the A53T familial mutant form of human $\alpha$-syn were injected with LPS. When compared to WT mice, only the transgenic mice displayed a persistent neuroinflammation, chronic progressive nigrostriatal degeneration, accumulation of aggregated, nitrated $\alpha$-syn, and formation of LB like inclusions in nigral neurons. In addition, a treatment with two inhibitors of inducible NOS and NADPH oxidase blocked the neurodegeneration in LPS-injected transgenic mice [156].

So how can we interpret the accumulating data on geneenvironment interactions in mouse models of PD? Although literature findings are not always consistent, most point towards a consensus, where parkin, PINK1, and DJ-1 are important in protecting the neurons in the face of oxidative damage, whereas $\alpha$-syn seems to play an intriguingly dual role, enhancing the sensitivity to oxidative stress in conditions of overexpression, and decreasing the sensitivity to oxidative stress in conditions of deletion (Figure 2). Altogether, there is significant evidence confirming that geneenvironment interactions are relevant to PD pathogenesis, and that the above-mentioned PD genes are involved in pathways linking oxidative stress to cell survival.

\section{Concluding Remarks}

Recent advances in understanding the genetics of PD in humans, as well as the use of animal models of PD, have enabled us to make important steps not only in identifying factors involved in the pathogenesis, but also in determining how these factors interact [9]. However, the lack of a clear picture regarding the pathogenesis of $\mathrm{PD}$ means that rational neuroprotective therapies are still missing. Therefore, there is currently an urgent need for the elucidation of the exact molecular architecture behind the pathogenesis of $\mathrm{PD}$, in order to provide sound bases for a rational pharmacological intervention that can prevent, slow down, or stop the progressive neuronal degeneration. 


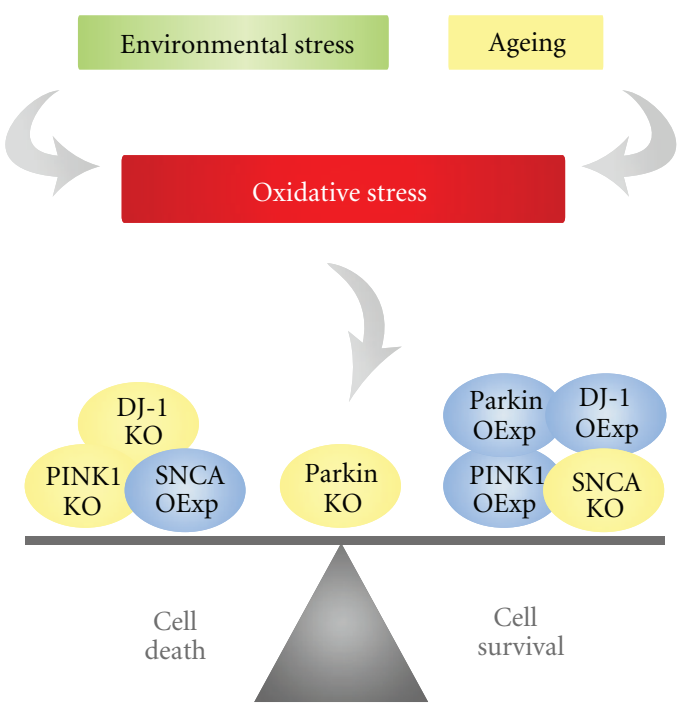

FIGURE 2: Gene-environment interactions involving oxidative stress in genetic mouse models of PD. Environmental factors and ageing converge to induce cellular oxidative stress, and this can either lead to cell death, or cell survival, based on genetic background. As shown in various gene-environment interaction studies in mouse models of PD (see Table 3), the PD genes can tilt the balance either to cell death, or to cell survival, thereby modulating the survival of the neurons following oxidative damage. Abbreviations: KO: knockout, OExp: overexpression.

Not only the genetic findings, but also compelling data from epidemiological studies, indicate that PD is a strong candidate for studying gene-environment interactions [167]. The susceptibility of an individual to develop PD probably involves a complex interplay of genetic factors, environmental factors and ageing, with the consequence that within a population of individuals with $\mathrm{PD}$, considerable heterogeneity exists with regard to function of specific cellular pathways [9]. Despite the importance of this interplay, not much data are available on gene-environment interaction studies in animal models of PD. Although different genetic animal models of PD have failed to fully reproduce the PD pathology, they still provide an interesting platform to investigate the role of the different PD-associated genes on the integrity of the nigrostriatal dopaminergic and other neuronal systems. The exposure of animal models to external stressors may accelerate the disease process and distinguish them more from animal models that are kept in well-controlled laboratory conditions away from stressors. We suggest that challenging genetically engineered animal models of PD might be of important value to predict the influences of the genetic alterations on the readouts. Furthermore, most of the studies that characterize animal models of PD only take into account the phenotype of the disease, without considering presymptomatic manifestations of PD. Investigating pre-symptomatic aspects, such as oxidative stress, might allow a more in-depth characterization of animal models.

Since biomarkers are important tools to monitor the disease status of an individual, efforts should be made to develop, standardize, and harmonize the different techniques to allow experimental studies to be performed across different centers ensuring power, allowing replication, and increasing their successfulness. Several promising approaches for PD biomarkers, such as proteomics, are heading towards promising directions (reviewed in [168-171]. Furthermore, it should be emphasized that the detection of more than one marker for oxidative stress is key, because a single marker might give misleading results [31]. More efforts should be made to extrapolate findings from PD patients to animal models and vice versa. Not only findings, but also the developed methodologies should be more applicable and/or easy adjustable for application in both animal models and PD patient. As mentioned above, it is wellknown that PD is a heterogeneous neurodegenerative disorder and results in the occurrence of a variety of symptoms, which forms the basis for classifying PD patients into different subtypes $[1,172,173]$. Since, the severity and type of the symptoms among these different subtypes may vary from patient to patient and may likely reflect distinct underlying etiologies, their identification and biochemical characterization may facilitate our understanding of their pathogenesis, which consequently may lead to more tailored therapies $[1,173]$. To our knowledge and after extensive literature research, no data have been found that investigated the different subtypes of PD in terms of impact of oxidative stress and pathogenesis.

Within this review, we focused on the complex interaction and complementary interrelationship between oxidative stress and neurodegeneration and the possible connection between oxidative stress and PD-associated genes. Although not always consistent, literature findings point to an important involvement of the PD genes parkin, PINK1, DJ-1, and SNCA in the oxidative stress pathogenic pathway and in protection against oxidative stress, confirming again that oxidative stress is an important pathogenic pathway in PD.

\section{Acknowledgments}

The authors wish to acknowledge the assistance of Mrs. R. Berckmans, Mr. G. De Smet, Mrs. C. De Rijck, and Mrs. R.M. Geens. This work was conducted with financial support from the Institute for the Promotion of Innovation by Science and Technology in Flanders (IWT) (IWT420) and of the Research Council from the Vrije Universiteit Brussel. M. Varçin is holder of a research grant from the IWT (IWT420). E. Bentea is a research fellow of the FWO-Vlaanderen.

\section{References}

[1] J. A. Obeso, M. C. Rodriguez-Oroz, C. G. Goetz et al., "Missing pieces in the Parkinson's disease puzzle," Nature Medicine, vol. 16, no. 6, pp. 653-661, 2010.

[2] D. Crosiers, J. Theuns, P. Cras, and C. Van Broeckhoven, "Parkinson disease: insights in clinical, genetic and pathological features of monogenic disease subtypes," Journal of Chemical Neuroanatomy, vol. 42, no. 2, pp. 131-141, 2011.

[3] C. Sundal, S. Fujioka, R. J. Uitti, and Z. K. Wszolek, "Autosomal dominant Parkinson's disease," Parkinsonism \& Related Disorders, vol. 18, supplement 1, pp. S7-S10, 2012. 
[4] H. Reichmann, "View point: etiology in Parkinson's disease. Dual hit or spreading intoxication," Journal of the Neurological Sciences, vol. 310, no. 1-2, pp. 9-11, 2011.

[5] S. Lesage and A. Brice, "Role of Mendelian genes in "sporadic" Parkinson's disease," Parkinsonism \& Related Disorders, vol. 18, supplement 1, pp. S66-S70, 2012.

[6] C. Taccioli, V. Maselli, J. Tegnér et al., “ParkDB: a Parkinson's disease gene expression database," Database, vol. 2011, article bar007, 2011.

[7] J. M. Shulman, P. L. De Jager, and M. B. Feany, "Parkinson's disease: genetics and pathogenesis," Annual Review of Pathology, vol. 6, pp. 193-222, 2011.

[8] C. J. Dunning et al., "Can Parkinson's disease pathology be propagated from one neuron to another?" Progress in Neurobiology. In press.

[9] C. Henchcliffe and F. M. Beal, "Mitochondrial biology and oxidative stress in Parkinson disease pathogenesis," Nature Clinical Practice Neurology, vol. 4, no. 11, pp. 600-609, 2008.

[10] H. Braak, K. Del Tredici, U. Rüb, R. A. I. De Vos, E. N. H. Jansen Steur, and E. Braak, "Staging of brain pathology related to sporadic Parkinson's disease," Neurobiology of Aging, vol. 24, no. 2, pp. 197-211, 2003.

[11] C. H. Hawkes, K. Del Tredici, and H. Braak, "A timeline for Parkinson's disease," Parkinsonism and Related Disorders, vol. 16, no. 2, pp. 79-84, 2010.

[12] A. H. V. Schapira, "Neurobiology and treatment of Parkinson's disease," Trends in Pharmacological Sciences, vol. 30, no. 1, pp. 41-47, 2009.

[13] F. Stocchi and C. W. Olanow, "Neuroprotection in Parkinson's disease: clinical trials," Annals of Neurology, vol. 53, no. 3, supplement, pp. S87-S99, 2003.

[14] T. A. Yacoubian and D. G. Standaert, "Targets for neuroprotection in Parkinson's disease," Biochimica et Biophysica Acta, vol. 1792, no. 7, pp. 676-687, 2009.

[15] D. Blum, S. Torch, N. Lambeng et al., "Molecular pathways involved in the neurotoxicity of 6-OHDA, dopamine and MPTP: contribution to the apoptotic theory in Parkinson's disease," Progress in Neurobiology, vol. 65, no. 2, pp. 135-172, 2001.

[16] K. Aoyama, M. Watabe, and T. Nakaki, "Regulation of neuronal glutathione synthesis," Journal of Pharmacological Sciences, vol. 108, no. 3, pp. 227-238, 2008.

[17] P. Jenner, "Oxidative stress in Parkinson's disease," Annals of Neurology, vol. 53, supplement 3, pp. S26-S36, 2003.

[18] S. Kawajiri, S. Saiki, S. Sato, and N. Hattori, "Genetic mutations and functions of PINK1," Trends in Pharmacological Sciences, vol. 32, no. 10, pp. 573-580, 2011.

[19] R. C. S. Seet, C. Y. J. Lee, E. C. H. Lim et al., "Oxidative damage in Parkinson disease: measurement using accurate biomarkers," Free Radical Biology and Medicine, vol. 48, no. 4, pp. 560-566, 2010.

[20] P. Jenner, "Oxidative mechanisms in nigral cell death in Parkinson's disease," Movement Disorders, vol. 13, no. 1, pp. 24-34, 1998.

[21] M. P. Smith and W. A. Cass, "Oxidative stress and dopamine depletion in an intrastriatal 6-hydroxydopamine model of Parkinson's disease," Neuroscience, vol. 144, no. 3, pp. 10571066, 2007.

[22] T. M. Dawson, H. S. Ko, and V. L. Dawson, "Genetic animal models of Parkinson's disease," Neuron, vol. 66, no. 5, pp. 646-661, 2010.

[23] M. J. Farrer, "Genetics of Parkinson disease: paradigm shifts and future prospects," Nature Reviews Genetics, vol. 7, no. 4, pp. 306-318, 2006.
[24] M. P. Horowitz and J. T. Greenamyre, "Gene-environment interactions in parkinson's disease: the importance of animal modeling," Clinical Pharmacology and Therapeutics, vol. 88, no. 4, pp. 467-474, 2010.

[25] K. J. Thomas and M. R. Cookson, "The role of PTENinduced kinase 1 in mitochondrial dysfunction and dynamics," International Journal of Biochemistry and Cell Biology, vol. 41, no. 10, pp. 2025-2035, 2009.

[26] D. J. Moore, A. B. West, V. L. Dawson, and T. M. Dawson, "Molecular pathophysiology of Parkinson's disease," Annual Review of Neuroscience, vol. 28, pp. 57-87, 2005.

[27] R. S. Akundi, L. Zhi, and H. Bueler, "PINK1 enhances insulin-like growth factor-1-dependent Akt signaling and protection against apoptosis," Neurobiology of Disease, vol. 45, no. 1, pp. 469-478, 2011.

[28] M. Diedrich, T. Kitada, G. Nebrich et al., "Brain region specific mitophagy capacity could contribute to selective neuronal vulnerability in Parkinson's disease," Proteome Science, vol. 9, p. 59, 2011.

[29] C. A. Gautier, T. Kitada, and J. Shen, "Loss of PINK1 causes mitochondrial functional defects and increased sensitivity to oxidative stress," Proceedings of the National Academy of Sciences of the United States of America, vol. 105, no. 32, pp. 11364-11369, 2008.

[30] E. Deas, H. Plun-Faureau, and N. W. Wood, "PINK1 function in health and disease," EMBO Molecular Medicine, vol. 1, no. 3, pp. 152-165, 2009.

[31] I. Dalle-Donne, D. Giustarini, R. Colombo, R. Rossi, and A. Milzani, "Protein carbonylation in human diseases," Trends in Molecular Medicine, vol. 9, no. 4, pp. 169-176, 2003.

[32] A. Toulouse and A. M. Sullivan, "Progress in Parkinson's disease-Where do we stand?" Progress in Neurobiology, vol. 85, no. 4, pp. 376-392, 2008.

[33] K. Cui, X. Luo, K. Xu, and M. R. Ven Murthy, "Role of oxidative stress in neurodegeneration: recent developments in assay methods for oxidative stress and nutraceutical antioxidants," Progress in Neuro-Psychopharmacology and Biological Psychiatry, vol. 28, no. 5, pp. 771-799, 2004.

[34] Y. Gilgun-Sherki, E. Melamed, and D. Offen, "Oxidative stress induced-neurodegenerative diseases: the need for antioxidants that penetrate the blood brain barrier," Neuropharmacology, vol. 40, no. 8, pp. 959-975, 2001.

[35] R. B. Mythri, C. Venkateshappa, G. Harish et al., "Evaluation of Markers of oxidative stress, antioxidant function and astrocytic proliferation in the striatum and frontal cortex of Parkinson's disease brains," Neurochemical Research, vol. 36, no. 8, pp. 1452-1463, 2011.

[36] J. K. Andersen, "Oxidative stress in neurodegeneration: cause or consequence?" Nature Medicine, vol. 10, pp. S18-S25, 2004.

[37] H. E. de Vries, M. Witte, D. Hondius et al., "Nrf2-induced antioxidant protection: a promising target to counteract ROS-mediated damage in neurodegenerative disease?" Free Radical Biology and Medicine, vol. 45, no. 10, pp. 1375-1383, 2008.

[38] S. Fahn and G. Cohen, "The oxidant stress hypothesis in Parkinson's disease: evidence supporting it," Annals of Neurology, vol. 32, no. 6, pp. 804-812, 1992.

[39] M. B. Youdim, N. Drigues, and S. Mandel, "Oxidative stress indices in Parkinson's disease: biochemical determination," Methods in Molecular Medicine, vol. 62, pp. 137-153, 2001.

[40] T. Ilic, M. Jovanović, A. Jovicić, and M. Tomović, "Oxidative stress and Parkinson's disease," Vojnosanitetski Pregled, vol. 55, no. 5, pp. 463-468, 1998. 
[41] M. Vinish, A. Anand, and S. Prabhakar, "Altered oxidative stress levels in Indian Parkinson's disease patients with PARK2 mutations," Acta Biochimica Polonica, vol. 58, no. 2, pp. 165-169, 2011.

[42] M. L. Selley, "(E)-4-Hydroxy-2-nonenal may be involved in the pathogenesis of Parkinson's disease," Free Radical Biology and Medicine, vol. 25, no. 2, pp. 169-174, 1998.

[43] C. Buhmann, S. Arlt, A. Kontush et al., "Plasma and CSF markers of oxidative stress are increased in Parkinson's disease and influenced by antiparkinsonian medication," Neurobiology of Disease, vol. 15, no. 1, pp. 160-170, 2004.

[44] P. del Hoyo, A. García-Redondo, F. de Bustos et al., "Oxidative stress in skin fibroblasts cultures from patients with Parkinson's disease," BMC Neurology, vol. 10, p. 95, 2011.

[45] B. Byers, B. Cord, H. N. Nguyen et al., "SNCA triplication Parkinson's patient's iPSC-derived DA neurons accumulate alpha-synuclein and are susceptible to oxidative stress," PLoS ONE, vol. 6, no. 11, Article ID e26159, 2011.

[46] F. P. Bellinger, M. T. Bellinger, L. A. Seale et al., "Glutathione peroxidase 4 is associated with neuromelanin in substantia nigra and dystrophic axons in putamen of Parkinson's brain," Molecular Neurodegeneration, vol. 6, no. 1, article 8, 2011.

[47] L. Migliore and F. Coppedè, "Environmental-induced oxidative stress in neurodegenerative disorders and aging," Mutation Research, vol. 674, no. 1-2, pp. 73-84, 2009.

[48] T. Müller and S. Muhlack, "Cysteinyl-glycine reduction as marker for levodopa-induced oxidative stress in Parkinson's disease patients," Movement Disorders, vol. 26, no. 3, pp. 543546, 2011.

[49] T. Müller, C. Jugel, R. Ehret et al., "Elevation of total homocysteine levels in patients with Parkinson's disease treated with duodenal levodopa/carbidopa gel," Journal of Neural Transmission, vol. 118, no. 9, pp. 1329-1333, 2011.

[50] A. H. V. Schapira, "Present and future drug treatment for Parkinson's disease," Journal of Neurology, Neurosurgery and Psychiatry, vol. 76, no. 11, pp. 1472-1478, 2005.

[51] K. J. Barnham, C. L. Masters, and A. I. Bush, "Neurodegenerative diseases and oxidatives stress," Nature Reviews Drug Discovery, vol. 3, no. 3, pp. 205-214, 2004.

[52] C. Mytilineou, B. C. Kramer, and J. A. Yabut, "Glutathione depletion and oxidative stress," Parkinsonism and Related Disorders, vol. 8, no. 6, pp. 385-387, 2002.

[53] R. A. Roberts, R. A. Smith, S. Safe, C. Szabo, R. B. Tjalkens, and F. M. Robertson, "Toxicological and pathophysiological roles of reactive oxygen and nitrogen species," Toxicology, vol. 276, no. 2, pp. 85-94, 2010.

[54] G. Stefanoni, G. Sala, and L. Tremolizzo, "Alpha-synuclein, oxidative stress and autophagy failure: dangerous liaisons in dopaminergic neurodegeneration," in Etiology and Pathophysiology of Parkinson's Disease, A. Q. Rana, Ed., InTech, 2011.

[55] D. M. Crabtree and J. Zhang, "Genetically engineered mouse models of Parkinson's disease," Brain Research Bulletin. In press.

[56] J. Lotharius and P. Brundin, "Pathogenesis of Parkinson's disease: dopamine, vesicles and alpha-synuclein," Nature reviews. Neuroscience, vol. 3, no. 12, pp. 932-942, 2002.

[57] A. H. Schapira, "Mitochondrial pathology in Parkinson's disease," Mount Sinai Journal of Medicine, vol. 78, no. 6, pp. 872-881, 2011.

[58] A. H. V. Schapira and M. Gegg, "Mitochondrial contribution to parkinson's disease pathogenesis," Parkinson's Disease, vol. 2011, Article ID 159160, 7 pages, 2011.
[59] R. K. Chaturvedi and M. F. Beal, "Mitochondrial approaches for neuroprotection," Annals of the New York Academy of Sciences, vol. 1147, pp. 395-412, 2008.

[60] R. H. Swerdlow, J. K. Parks, S. W. Miller et al., "Origin and functional consequences of the complex I defect in Parkinson's disease," Annals of Neurology, vol. 40, no. 4, pp. 663-671, 1996.

[61] S. Fulda, L. Galluzzi, and G. Kroemer, "Targeting mitochondria for cancer therapy," Nature Reviews Drug Discovery, vol. 9, no. 6, pp. 447-464, 2010.

[62] M. Dumont and M. F. Beal, "Neuroprotective strategies involving ROS in Alzheimer disease," Free Radical Biology and Medicine, vol. 51, no. 5, pp. 1014-1026, 2011.

[63] P. M. Abou-Sleiman, M. M. K. Muqit, and N. W. Wood, "Expanding insights of mitochondrial dysfunction in Parkinson's disease," Nature Reviews Neuroscience, vol. 7, no. 3, pp. 207-219, 2006.

[64] J. A. Petko, N. Kabbani, C. Frey et al., "Proteomic and functional analysis of NCS-1 binding proteins reveals novel signaling pathways required for inner ear development in zebrafish," BMC Neuroscience, vol. 10, article 27, 2009.

[65] S. Gandhi, A. Wood-Kaczmar, Z. Yao et al., "PINK1associated Parkinson's disease is caused by neuronal vulnerability to calcium-induced cell death," Molecular Cell, vol. 33, no. 5, pp. 627-638, 2009.

[66] M. L. Block, L. Zecca, and J. S. Hong, "Microgliamediated neurotoxicity: uncovering the molecular mechanisms," Nature Reviews Neuroscience, vol. 8, no. 1, pp. 57-69, 2007.

[67] A. G. Ceulemans, T. Zgavc, R. Kooijman, S. Hachimi-Idrissi, S. Sarre, and Y. Michotte, "The dual role of the neuroinflammatory response after ischemic stroke: modulatory effects of hypothermia," Journal of Neuroinflammation, vol. 7, article 74,2010

[68] C. Chéret, A. Gervais, A. Lelli et al., "Neurotoxic activation of microglia is promoted by a Nox1-dependent NADPH oxidase," Journal of Neuroscience, vol. 28, no. 46, pp. 1203912051, 2008.

[69] P. S. Whitton, "Inflammation as a causative factor in the aetiology of Parkinson's disease," British Journal of Pharmacology, vol. 150, no. 8, pp. 963-976, 2007.

[70] C. M. Long-Smith, A. M. Sullivan, and Y. M. Nolan, "The influence of microglia on the pathogenesis of Parkinson's disease," Progress in Neurobiology, vol. 89, no. 3, pp. 277-287, 2009.

[71] L. Qian, P. M. Flood, and J. S. Hong, "Neuroinflammation is a key player in Parkinson's disease and a prime target for therapy," Journal of Neural Transmission, vol. 117, no. 8, pp. 971-979, 2010.

[72] J. S. Beckman, T. W. Beckman, J. Chen, P. A. Marshall, and B. A. Freeman, "Apparent hydroxyl radical production by peroxynitrite: implications for endothelial injury from nitric oxide and superoxide," Proceedings of the National Academy of Sciences of the United States of America, vol. 87, no. 4, pp. 1620-1624, 1990.

[73] T. Münzel and J. F. Keaney, "Are ACE inhibitors a "magic bullet" against oxidative stress?" Circulation, vol. 104, no. 13, pp. 1571-1574, 2001.

[74] V. Anantharam, S. Kaul, C. Song, A. Kanthasamy, and A. G. Kanthasamy, "Pharmacological inhibition of neuronal NADPH oxidase protects against 1-methyl-4phenylpyridinium (MPP+)-induced oxidative stress and apoptosis in mesencephalic dopaminergic neuronal cells," NeuroToxicology, vol. 28, no. 5, pp. 988-997, 2007. 
[75] B. Mertens, P. Vanderheyden, Y. Michotte, and S. Sarre, "The role of the central renin-angiotensin system in Parkinson's disease," Journal of the Renin-Angiotensin-Aldosterone System, vol. 11, no. 1, pp. 49-56, 2010.

[76] B. Mertens, M. Varcin, Y. Michotte, and S. Sarre, "The neuroprotective action of candesartan is related to interference with the early stages of 6-hydroxydopamine-induced dopaminergic cell death," European Journal of Neuroscience, vol. 34, no. 7, pp. 1141-1148, 2011.

[77] V. H. Perry, J. A. R. Nicoll, and C. Holmes, "Microglia in neurodegenerative disease," Nature Reviews Neurology, vol. 6, no. 4, pp. 193-201, 2010.

[78] T. M. Dawson and V. L. Dawson, "Molecular pathways of neurodegeneration in Parkinson's disease," Science, vol. 302, no. 5646, pp. 819-822, 2003.

[79] K. J. A. Davies, "Oxidative stress, antioxidant defenses, and damage removal, repair, and replacement systems," IUBMB Life, vol. 50, no. 4-5, pp. 279-289, 2000.

[80] T. Finkel and N. J. Holbrook, "Oxidants, oxidative stress and the biology of ageing," Nature, vol. 408, no. 6809, pp. 239$247,2000$.

[81] J. Clark and D. K. Simon, "Transcribe to survive: transcriptional control of antioxidant defense programs for neuroprotection in parkinson's disease," Antioxidants and Redox Signaling, vol. 11, no. 3, pp. 509-528, 2009.

[82] J. G. Scandalios, "Oxidative stress: molecular perception and transduction of signals triggering antioxidant gene defenses," Brazilian Journal of Medical and Biological Research, vol. 38, no. 7, pp. 995-1014, 2005.

[83] V. Calabrese, E. Guagliano, M. Sapienza et al., "Redox regulation of cellular stress response in aging and neurodegenerative disorders: role of vitagenes," Neurochemical Research, vol. 32, no. 4-5, pp. 757-773, 2007.

[84] G. Twig, A. Elorza, A. J. A. Molina et al., "Fission and selective fusion govern mitochondrial segregation and elimination by autophagy," EMBO Journal, vol. 27, no. 2, pp. 433-446, 2008.

[85] T. Tatsuta and T. Langer, "Quality control of mitochondria: protection against neurodegeneration and ageing," EMBO Journal, vol. 27, no. 2, pp. 306-314, 2008.

[86] C. Vives-Bauza and S. Przedborski, "Mitophagy: the latest problem for Parkinson's disease," Trends in Molecular Medicine, vol. 17, no. 3, pp. 158-165, 2011.

[87] R. J. Youle and D. P. Narendra, "Mechanisms of mitophagy," Nature Reviews Molecular Cell Biology, vol. 12, no. 1, pp. 914, 2011.

[88] M. F. Beal, "Parkinson's disease: a model dilemma," Nature, vol. 466, no. 7310, pp. S8-S10, 2010.

[89] H. L. Melrose, S. J. Lincoln, G. M. Tyndall, and M. J. Farrer, "Parkinson's disease: a rethink of rodent models," Experimental Brain Research, vol. 173, no. 2, pp. 196-204, 2006.

[90] S. Duty and P. Jenner, "Animal models of Parkinson's disease: a source of novel treatments and clues to the cause of the disease," British Journal of Pharmacology, vol. 164, no. 4, pp. 1357-1391, 2011.

[91] M. A. Gama Sosa, R. de Gasperi, and G. A. Elder, "Modeling human neurodegenerative diseases in transgenic systems," Human Genetics, vol. 131, no. 4, pp. 535-563, 2012.

[92] C. Fernandes and Y. Rao, "Genome-wide screen for modifiers of Parkinson's disease genes in Drosophila," Molecular Brain, vol. 4, no. 1, article 17, 2011.

[93] L. Chen, B. Cagniard, T. Mathews et al., "Age-dependent motor deficits and dopaminergic dysfunction in DJ-1 null mice," Journal of Biological Chemistry, vol. 280, no. 22, pp. 21418-21426, 2005.
[94] E. Andres-Mateos, C. Perier, L. Zhang et al., "DJ-1 gene deletion reveals that DJ-1 is an atypical peroxiredoxin-like peroxidase," Proceedings of the National Academy of Sciences of the United States of America, vol. 104, no. 37, pp. 1480714812, 2007.

[95] H. Yamaguchi and J. Shen, "Absence of dopaminergic neuronal degeneration and oxidative damage in aged DJ-Ideficient mice," Molecular Neurodegeneration, vol. 2, no. 1, article 10, 2007.

[96] J. N. Guzman, J. Sanchez-Padilla, D. Wokosin et al., “Oxidant stress evoked by pacemaking in dopaminergic neurons is attenuated by DJ-1," Nature, vol. 468, no. 7324, pp. 696-700, 2010.

[97] I. Irrcher, H. Aleyasin, E. L. Seifert et al., "Loss of the Parkinson's disease-linked gene DJ-1 perturbs mitochondrial dynamics," Human Molecular Genetics, vol. 19, no. 19, pp. 3734-3746, 2010.

[98] F. A. Perez, W. R. Curtis, and R. D. Palmiter, "Parkindeficient mice are not more sensitive to 6-hydroxydopamine or methamphetamine neurotoxicity," BMC Neuroscience, vol. 6, article 71, 2005.

[99] J. M. Itier, P. Ibáñez, M. A. Mena et al., "Parkin gene inactivation alters behaviour and dopamine neurotransmission in the mouse," Human Molecular Genetics, vol. 12, no. 18, pp. 2277-2291, 2003.

[100] J. J. Palacino, D. Sagi, M. S. Goldberg et al., "Mitochondrial dysfunction and oxidative damage in parkin-deficient mice," Journal of Biological Chemistry, vol. 279, no. 18, pp. 1861418622, 2004.

[101] M. Periquet, O. Corti, S. Jacquier, and A. Brice, "Proteomic analysis of parkin knockout mice: alterations in energy metabolism, protein handling and synaptic function," Journal of Neurochemistry, vol. 95, no. 5, pp. 1259-1276, 2005.

[102] J. A. Rodríguez-Navarro, M. J. Casarejos, J. Menéndez et al., "Mortality, oxidative stress and tau accumulation during ageing in parkin null mice," Journal of Neurochemistry, vol. 103, no. 1, pp. 98-114, 2007.

[103] X. H. Lu, S. M. Fleming, B. Meurers et al., "Bacterial artificial chromosome transgenic mice expressing a truncated mutant parkin exhibit age-dependent hypokinetic motor deficits, dopaminergic neuron degeneration, and accumulation of proteinase k-resistant $\alpha$-Synuclein," Journal of Neuroscience, vol. 29, no. 7, pp. 1962-1976, 2009.

[104] R. S. Akundi, Z. Huang, J. Eason et al., "Increased mitochondrial calcium sensitivity and abnormal expression of innate immunity genes precede dopaminergic defects in Pink1deficient mice," PLoS ONE, vol. 6, no. 1, Article ID e16038, 2011.

[105] F. Billia, L. Hauck, F. Konecny, V. Rao, J. Shen, and T. W. Mak, "PTEN-inducible kinase 1 (PINK1)/Park6 is indispensable for normal heart function," Proceedings of the National Academy of Sciences of the United States of America, vol. 108, no. 23, pp. 9572-9577, 2011.

[106] P. Klivenyi, D. Siwek, G. Gardian et al., "Mice lacking alphasynuclein are resistant to mitochondrial toxins," Neurobiology of Disease, vol. 21, no. 3, pp. 541-548, 2006.

[107] M. Neumann, P. J. Kahle, B. I. Giasson et al., "Misfolded proteinase K-resistant hyperphosphorylated $\alpha$-synuclein in aged transgenic mice with locomotor deterioration and in human $\alpha$-synucleinopathies," Journal of Clinical Investigation, vol. 110, no. 10, pp. 1429-1439, 2002.

[108] H. F. Poon, M. Frasier, N. Shreve, V. Calabrese, B. Wolozin, and D. A. Butterfield, "Mitochondrial associated metabolic 
proteins are selectively oxidized in A30P $\alpha$-synuclein transgenic mice-a model of familial Parkinson's disease," Neurobiology of Disease, vol. 18, no. 3, pp. 492-498, 2005.

[109] B. I. Giasson, J. E. Duda, S. M. Quinn, B. Zhang, J. Q. Trojanowski, and V. M. Y. Lee, "Neuronal $\alpha$-synucleinopathy with severe movement disorder in mice expressing A53T human $\alpha$-synuclein,” Neuron, vol. 34, no. 4, pp. 521-533, 2002.

[110] L. J. Martin, Y. Pan, A. C. Price et al., "Parkinson's disease $\alpha$-synuclein transgenic mice develop neuronal mitochondrial degeneration and cell death," Journal of Neuroscience, vol. 26, no. 1, pp. 41-50, 2006.

[111] R. M. Miller, G. L. Kiser, T. Kaysser-Kranich et al., "Wild-type and mutant $\alpha$-synuclein induce a multi-component gene expression profile consistent with shared pathophysiology in different transgenic mouse models of PD," Experimental Neurology, vol. 204, no. 1, pp. 421-432, 2007.

[112] A. O. Koob, K. Ubhi, J. F. Paulsson et al., "Lovastatin ameliorates $\alpha$-synuclein accumulation and oxidation in transgenic mouse models of $\alpha$-synucleinopathies," Experimental Neurology, vol. 221, no. 2, pp. 267-274, 2010.

[113] K. A. Brandis, I. F. Holmes, S. J. England, N. Sharma, L. Kukreja, and S. K. DebBurman, " $\alpha$-synuclein fission yeast model: concentration-dependent aggregation without plasma membrane localization or toxicity," Journal of Molecular Neuroscience, vol. 28, no. 2, pp. 179-192, 2006.

[114] F. A. Perez and R. D. Palmiter, "Parkin-deficient mice are not a robust model of parkinsonism," Proceedings of the National Academy of Sciences of the United States of America, vol. 102, no. 6, pp. 2174-2179, 2005.

[115] T. Kitada, A. Pisani, M. Karouani et al., "Impaired dopamine release and synaptic plasticity in the striatum of Parkin-/mice," Journal of Neurochemistry, vol. 110, no. 2, pp. 613-621, 2009.

[116] J. H. Son, H. Kawamata, M. S. Yoo et al., "Neurotoxicity and behavioral deficits associated with Septin 5 accumulation in dopaminergic neurons," Journal of Neurochemistry, vol. 94, no. 4, pp. 1040-1053, 2005.

[117] S. Sato, T. Chiba, S. Nishiyama et al., "Decline of striatal dopamine release in parkin-deficient mice shown by ex vivo autoradiography," Journal of Neuroscience Research, vol. 84, no. 6, pp. 1350-1357, 2006.

[118] R. Von Coelln, B. Thomas, J. M. Savitt et al., "Loss of locus coeruleus neurons and reduced startle in parkin null mice," Proceedings of the National Academy of Sciences of the United States of America, vol. 101, no. 29, pp. 10744-10749, 2004.

[119] X. Wang, D. Winter, G. Ashrafi et al., "PINK1 and Parkin target Miro for phosphorylation and degradation to arrest mitochondrial motility," Cell, vol. 147, no. 4, pp. 893-906, 2011.

[120] V. A. Morais, P. Verstreken, A. Roethig et al., "Parkinson's disease mutations in PINK1 result in decreased Complex I activity and deficient synaptic function," EMBO Molecular Medicine, vol. 1, no. 2, pp. 99-111, 2009.

[121] T. Kitada, A. Pisani, D. R. Porter et al., "Impaired dopamine release and synaptic plasticity in the striatum of PINK1deficient mice," Proceedings of the National Academy of Sciences of the United States of America, vol. 104, no. 27, pp. 11441-11446, 2007.

[122] S. Gispert, F. Ricciardi, A. Kurz et al., "Parkinson phenotype in aged PINK1-deficient mice is accompanied by progressive mitochondrial dysfunction in absence of neurodegeneration," PLoS ONE, vol. 4, no. 6, Article ID e5777, 2009.
[123] H. Zhou, B. H. Falkenburger, J. B. Schulz, K. Tieu, Z. Xu, and G. X. Xu, "Silencing of the Pink1 gene expression by conditional RNAi does not induce dopaminergic neuron death in mice," International Journal of Biological Sciences, vol. 3, no. 4, pp. 242-250, 2007.

[124] R. D. Mills, C. H. Sim, S. S. Mok, T. D. Mulhern, J. G. Culvenor, and H. C. Cheng, "Biochemical aspects of the neuroprotective mechanism of PTEN-induced kinase-1 (PINK1)," Journal of Neurochemistry, vol. 105, no. 1, pp. 1833, 2008.

[125] A. Wood-Kaczmar, S. Gandhi, Z. Yao et al., "PINK1 is necessary for long term survival and mitochondrial function in human dopaminergic neurons," PLoS ONE, vol. 3, no. 6, Article ID e2455, 2008.

[126] M. E. Gegg, J. M. Cooper, A. H. V. Schapira, and J. W. Taanman, "Silencing of PINK1 expression affects mitochondrial DNA and oxidative phosphorylation in DOPAMINERGIC cells," PLoS ONE, vol. 4, no. 3, Article ID e4756, 2009.

[127] N. Kabbani, L. Negyessy, R. Lin, P. Goldman-Rakic, and R. Levenson, "Interaction with neuronal calcium sensor NCS1 mediates desensitization of the D2 dopamine receptor," Journal of Neuroscience, vol. 22, no. 19, pp. 8476-8486, 2002.

[128] O. Pongs, J. Lindemeier, X. R. Zhu et al., "Frequenin-a novel calcium-binding protein that modulates synaptic efficacy in the Drosophila nervous system," Neuron, vol. 11, no. 1, pp. 15-28, 1993.

[129] T. Hatano, S. I. Kubo, S. Sato, and N. Hattori, "Pathogenesis of familial Parkinson's disease: new insights based on monogenic forms of Parkinson's disease," Journal of Neurochemistry, vol. 111, no. 5, pp. 1075-1093, 2009.

[130] J. H. Pogson, R. M. Ivatt, and A. J. Whitworth, "Molecular mechanisms of PINK1-related neurodegeneration," Current Neurology and Neuroscience Reports, vol. 11, no. 3, pp. 283290, 2011.

[131] A. Pilsl and K. F. Winklhofer, "Parkin, PINK1 and mitochondrial integrity: emerging concepts of mitochondrial dysfunction in Parkinson's disease," Acta Neuropathologica, vol. 2, pp. 173-188, 2012.

[132] D. P. Narendra, S. M. Jin, A. Tanaka et al., "PINK1 is selectively stabilized on impaired mitochondria to activate Parkin," PLoS Biology, vol. 8, no. 1, Article ID e1000298, 2010.

[133] T. Kitada, Y. Tong, C. A. Gautier, and J. Shen, "Absence of nigral degeneration in aged parkin/DJ-1/PINK1 triple knockout mice," Journal of Neurochemistry, vol. 111, no. 3, pp. 696-702, 2009.

[134] J. A. Klein and S. L. Ackerman, "Oxidative stress, cell cycle, and neurodegeneration," Journal of Clinical Investigation, vol. 111, no. 6, pp. 785-793, 2003.

[135] T. Hatano and N. Hattori, "Etiology and pathogenesis of Parkinson's disease," in Etiology and Pathophysiology of Parkinson's Disease, A. Q. Rana, Ed., InTech, 2011.

[136] A. R. Chade, M. Kasten, and C. M. Tanner, "Nongenetic causes of Parkinson's disease," Journal of Neural Transmission, no. 70, supplement, pp. 147-151, 2006.

[137] S. C. Marques, C. R. Oliveira, C. M. Pereira, and T. F. Outeiro, "Epigenetics in neurodegeneration: a new layer of complexity," Progress in Neuro-Psychopharmacology \& Biological Psychiatry, vol. 35, no. 2, pp. 348-355, 2011.

[138] O. Babenko, I. Kovalchuk, and G. A. Metz, "Epigenetic programming of neurodegenerative diseases by an adverse environment," Brain Research, vol. 1444, pp. 96-111, 2012.

[139] F. Blandini, M. T. Armentero, and E. Martignoni, "The 6hydroxydopamine model: news from the past," Parkinsonism and Related Disorders, vol. 14, no. 2, pp. S124-S129, 2008. 
[140] R. H. Kim, P. D. Smith, H. Aleyasin et al., "Hypersensitivity of DJ-1-deficient mice to 1-methyl-4-phenyl-1,2,3,6- tetrahydropyrindine (MPTP) and oxidative stress," Proceedings of the National Academy of Sciences of the United States of America, vol. 102, no. 14, pp. 5215-5220, 2005.

[141] A. B. Manning-Bog et al., "Increased vulnerability of nigrostriatal terminals in DJ-1-deficient mice is mediated by the dopamine transporter," Neurobiology of Disease, vol. 27, no. 2, pp. 141-150, 2007.

[142] J. C. Paterna, A. Leng, E. Weber, J. Feldon, and H. Büeler, "DJ-1 and parkin modulate dopamine-dependent behavior and inhibit MPTP-induced nigral dopamine neuron loss in mice," Molecular Therapy, vol. 15, no. 4, pp. 698-704, 2007.

[143] H. Zhou, C. Huang, J. Tong, and X. G. Xia, "Early exposure to paraquat sensitizes dopaminergic neurons to subsequent silencing of PINK1 gene expression in mice," International Journal of Biological Sciences, vol. 7, no. 8, pp. 1180-1187, 2011.

[144] M. E. Haque, K. J. Thomas, C. D’Souza et al., "Cytoplasmic Pink1 activity protects neurons from dopaminergic neurotoxin MPTP," Proceedings of the National Academy of Sciences of the United States of America, vol. 105, no. 5, pp. 1716-1721, 2008.

[145] W. Dauer, N. Kholodilov, M. Vila et al., "Resistance of $\alpha$ synuclein null mice to the parkinsonian neurotoxin MPTP," Proceedings of the National Academy of Sciences of the United States of America, vol. 99, no. 22, pp. 14524-14529, 2002.

[146] O. M. Schlüter, F. Fornai, M. G. Alessandrí et al., "Role of $\alpha$ synuclein in 1-methyl-4-phenyl-1,2,3,6-tetrahydropyridineinduced Parkinsonism in mice," Neuroscience, vol. 118, no. 4, pp. 985-1002, 2003.

[147] R. E. Drolet, B. Behrouz, K. J. Lookingland, and J. L. Goudreau, "Mice lacking $\alpha$-synuclein have an attenuated loss of striatal dopamine following prolonged chronic MPTP administration," NeuroToxicology, vol. 25, no. 5, pp. 761-769, 2004.

[148] D. C. Robertson, O. Schmidt, N. Ninkina, P. A. Jones, J. Sharkey, and V. L. Buchman, "Developmental loss and resistance to MPTP toxicity of dopaminergic neurones in substantia nigra pars compacta of $\gamma$-synuclein, $\alpha$-synuclein and double $\alpha / \gamma$-synuclein null mutant mice," Journal of Neurochemistry, vol. 89, no. 5, pp. 1126-1136, 2004.

[149] F. Fornai, O. M. Schlüter, P. Lenzi et al., "Parkinson-like syndrome induced by continuous MPTP infusion: convergent roles of the ubiquitin-proteasome system and $\alpha$-synuclein," Proceedings of the National Academy of Sciences of the United States of America, vol. 102, no. 9, pp. 3413-3418, 2005.

[150] D. Alvarez-Fischer, C. Henze, C. Strenzke et al., "Characterization of the striatal 6-OHDA model of Parkinson's disease in wild type and $\alpha$-synuclein-deleted mice," Experimental Neurology, vol. 210, no. 1, pp. 182-193, 2008.

[151] S. Rathke-Hartlieb, P. J. Kahle, M. Neumann et al., "Sensitivity to MPTP is not increased in Parkinson' s diseaseassociated mutant $\alpha$-synuclein transgenic mice," Journal of Neurochemistry, vol. 77, no. 4, pp. 1181-1184, 2001.

[152] M. Nieto, F. J. Gil-Bea, E. Dalfó et al., "Increased sensitivity to MPTP in human $\alpha$-synuclein A30P transgenic mice," Neurobiology of Aging, vol. 27, no. 6, pp. 848-856, 2006.

[153] Z. Dong, B. Ferger, J. Feldon, and H. Büeler, "Overexpression of Parkinson's disease-associated $\alpha$-synucleinA53T by recombinant adeno-associated virus in mice does not increase the vulnerability of dopaminergic neurons to MPTP," Journal of Neurobiology, vol. 53, no. 1, pp. 1-10, 2002.
[154] W. H. Yu, Y. Matsuoka, I. Sziráki et al., "Increased dopaminergic neuron sensitivity to 1-methyl-4-phenyl1,2,3,6- tetrahydropyridine (MPTP) in transgenic mice expressing mutant A53T $\alpha$-synuclein," Neurochemical Research, vol. 33, no. 5, pp. 902-911, 2008.

[155] J. Peng, M. L. Oo, and J. K. Andersen, "Synergistic effects of environmental risk factors and gene mutations in Parkinson's disease accelerate age-related neurodegeneration," Journal of Neurochemistry, vol. 115, no. 6, pp. 1363-1373, 2010.

[156] H. M. Gao, F. Zhang, H. Zhou, W. Kam, B. Wilson, and J. S. Hong, "Neuroinflammation and $\alpha$-synuclein dysfunction potentiate each other, driving chronic progression of neurodegeneration in a mouse model of Parkinson's disease," Environmental Health Perspectives, vol. 119, no. 6, pp. 807814, 2011.

[157] P. O. Fernagut, C. B. Hutson, S. M. Fleming et al., "Behavioral and histopathological consequences of paraquat intoxication in mice: effects of $\alpha$-synuclein over-expression," Synapse, vol. 61, no. 12, pp. 991-1001, 2007.

[158] E. K. Richfield, M. J. Thiruchelvam, D. A. Cory-Slechta et al., "Behavioral and neurochemical effects of wild-type and mutated human $\alpha$-synuclein in transgenic mice," Experimental Neurology, vol. 175, no. 1, pp. 35-48, 2002.

[159] M. J. Thiruchelvam, J. M. Powers, D. A. Cory-Slechta, and E. K. Richfield, "Risk factors for dopaminergic neuron loss in human $\alpha$-synuclein transgenic mice," European Journal of Neuroscience, vol. 19, no. 4, pp. 845-854, 2004.

[160] N. M. Filipov, A. B. Norwood, and S. C. Sistrunk, "Strainspecific sensitivity to MPTP of C57BL/6 and BALB/c mice is age dependent," NeuroReport, vol. 20, no. 7, pp. 713-717, 2009.

[161] S. Grealish, B. Mattsson, P. Draxler, and A. Björklund, "Characterisation of behavioural and neurodegenerative changes induced by intranigral 6-hydroxydopamine lesions in a mouse model of Parkinson's disease," European Journal of Neuroscience, vol. 31, no. 12, pp. 2266-2278, 2010.

[162] R. Iancu, P. Mohapel, P. Brundin, and G. Paul, "Behavioral characterization of a unilateral 6-OHDA-lesion model of Parkinson's disease in mice," Behavioural Brain Research, vol. 162, no. 1, pp. 1-10, 2005.

[163] R. K. Schwarting, M. Sedelis, K. Hofele et al., "Straindependent recovery of open-field behavior and striatal dopamine deficiency in the mouse MPTP model of Parkinson's disease," Neurotoxicity Research, vol. 1, no. 1, pp. 41-56, 1999.

[164] M. Sedelis, K. Hofele, G. W. Auburger, S. Morgan, J. P. Huston, and R. K. W. Schwarting, "MPTP susceptibility in the mouse: behavioral, neurochemical, and histological analysis of gender and strain differences," Behavior Genetics, vol. 30, no. 3, pp. 171-182, 2000.

[165] M. Lee, D. H. Hyun, B. Halliwell, and P. Jenner, "Effect of the overexpression of wild-type or mutant $\alpha$-synuclein on cell susceptibility to insult," Journal of Neurochemistry, vol. 76, no. 4, pp. 998-1009, 2001.

[166] M. Orth, S. J. Tabrizi, C. Tomlinson et al., "G209A mutant alpha synuclein expression specifically enhances dopamine induced oxidative damage," Neurochemistry International, vol. 45, no. 5, pp. 669-676, 2004.

[167] T. H. Hamza, H. Chen, E. M. Hill-Burns et al., "Genomewide gene-environment study identifies glutamate receptor gene GRIN2A as a Parkinson's disease modifier gene via interaction with coffee," PLoS Genetics, vol. 7, no. 8, Article ID e1002237, 2011. 
[168] P. Lewitt, "Recent advances in CSF biomarkers for Parkinson's disease," Parkinsonism \& Related Disorders, vol. 18, supplement 1, pp. S49-S51, 2012.

[169] K. D. van Dijk, C. E. Teunissen, B. Drukarch et al., "Diagnostic cerebrospinal fluid biomarkers for Parkinson's disease: a pathogenetically based approach," Neurobiology of Disease, vol. 39, no. 3, pp. 229-241, 2010.

[170] M. Gerlach, W. Maetzler, K. Broich et al., "Biomarker candidates of neurodegeneration in Parkinson's disease for the evaluation of disease-modifying therapeutics," Journal of Neural Transmission, vol. 119, no. 1, pp. 39-52, 2011.

[171] T. Alberio and M. Fasano, "Proteomics in Parkinson's disease: an unbiased approach towards peripheral biomarkers and new therapies," Journal of Biotechnology, vol. 156, no. 4, pp. 325-337, 2011.

[172] R. C. Helmich, H. Mark, G. Deuschl et al., "Cerebral causes and consequences of parkinsonian resting tremor: a tale of two circuits?" Brain. In press.

[173] S. M. Van Rooden, F. Colas, P. Martínez-Martín et al., "Clinical subtypes of Parkinson's disease," Movement Disorders, vol. 26, no. 1, pp. 51-58, 2011. 


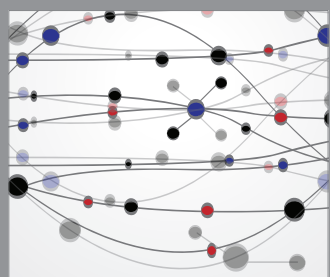

The Scientific World Journal
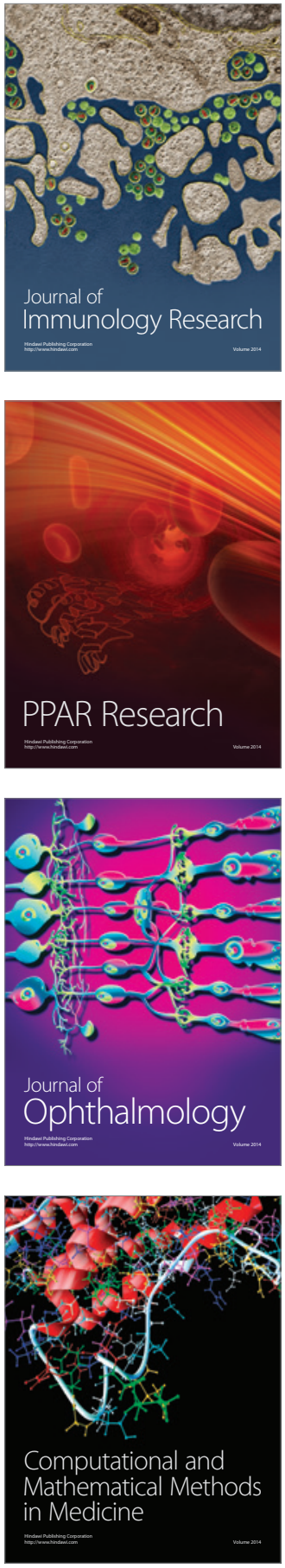

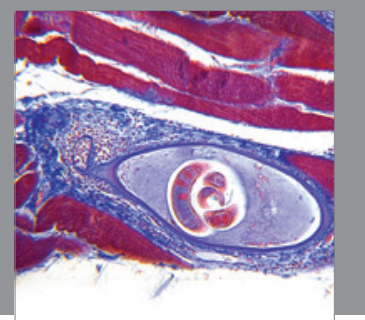

Gastroenterology

Research and Practice
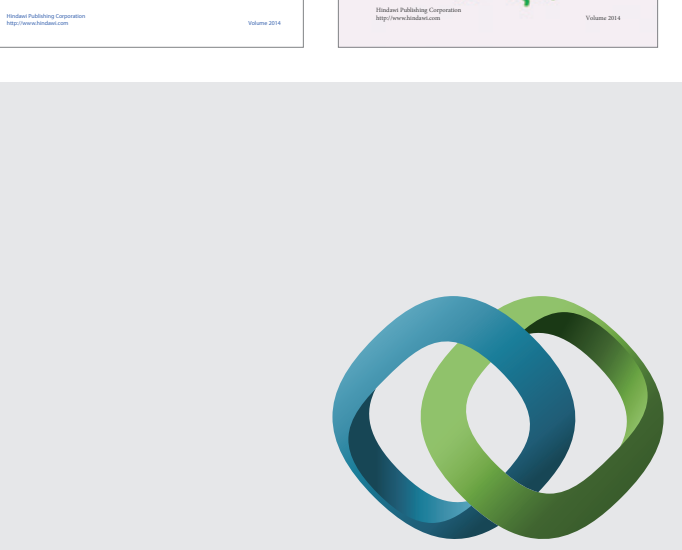

\section{Hindawi}

Submit your manuscripts at

http://www.hindawi.com
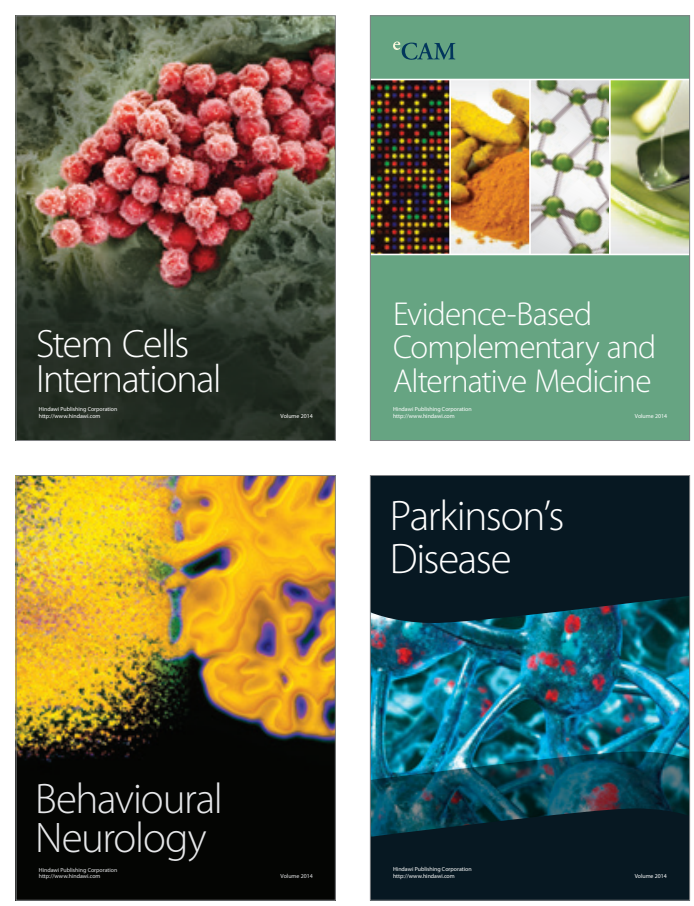

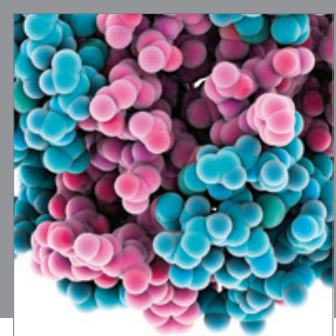

Journal of
Diabetes Research

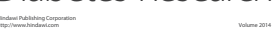

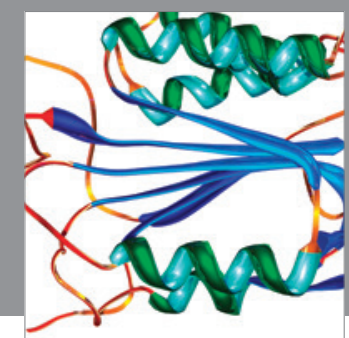

Disease Markers
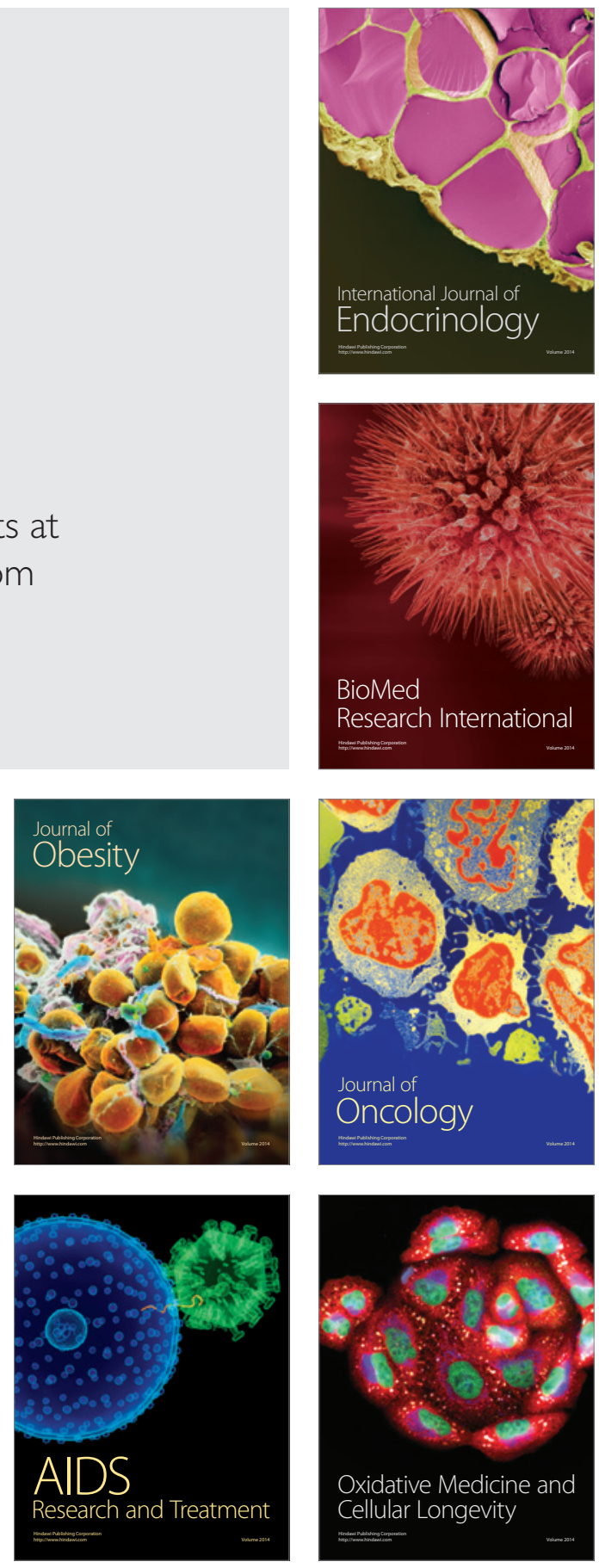